\title{
Conformal Subnets and Intermediate Subfactors
}

\author{
ROBERTO LONGO* \\ Dipartimento di Matematica \\ Università di Roma "Tor Vergata" \\ Via della Ricerca Scientifica, I-00133 Roma, Italy \\ e-mail: longo@mat.uniroma2.it
}

Dedicated to Rudolf Haag on the occasion of his eightieth birthday

\begin{abstract}
Given an irreducible local conformal net $\mathcal{A}$ of von Neumann algebras on $S^{1}$ and a finite-index conformal subnet $\mathcal{B} \subset \mathcal{A}$, we show that $\mathcal{A}$ is completely rational iff $\mathcal{B}$ is completely rational. In particular this extends a result of $\mathrm{F}$. Xu for the orbifold construction. By applying previous results of $\mathrm{Xu}$, many coset models turn out to be completely rational and the structure results in [27] hold. Our proofs are based on an analysis of the net inclusion $\mathcal{B} \subset \mathcal{A}$; among other things we show that, for a fixed interval $I$, every von Neumann algebra $\mathcal{R}$ intermediate between $\mathcal{B}(I)$ and $\mathcal{A}(I)$ comes from an intermediate conformal net $\mathcal{L}$ between $\mathcal{B}$ and $\mathcal{A}$ with $\mathcal{L}(I)=\mathcal{R}$. We make use of a theorem of Watatani (type II case) and Teruya and Watatani (type III case) on the finiteness of the set $\mathfrak{I}(\mathcal{N}, \mathcal{M})$ of intermediate subfactors in an irreducible inclusion of factors $\mathcal{N} \subset \mathcal{M}$ with finite Jones index $[\mathcal{M}: \mathcal{N}]$. We provide a unified proof of this result that gives in particular an explicit bound for the cardinality of $\mathfrak{I}(\mathcal{N}, \mathcal{M})$ which depends only on $[\mathcal{M}: \mathcal{N}]$.
\end{abstract}

\footnotetext{
${ }^{*}$ Supported in part by MURST and INDAM-GNAMPA.
} 


\section{Introduction}

Operator algebraic methods have been used to good effect in Conformal Quantum Field Theory, in particular in understanding general model independent structure (e.g. [6, 27, 16, 22, 23, 38]), in the analysis of concrete models (e.g. [5, 43, 44, 46]) and for applications in different contexts (e.g. [33]). In most cases it seems to be impossible to proceed by different methods.

Because of their relevance in different areas, among others Topological QFT and 3-manifold invariants, conformal models with a rational and modular representation theory have been the subject of much attention, also in the physical literature (cf. [14).

In [27 intrinsic, model independent conditions selecting a class of (local, irreducible) conformal nets $\mathcal{A}$ of von Neumann algebras on $S^{1}$ with the right rationality/modularity properties were given. $\mathcal{A}$ is completely rational if

1. $\mathcal{A}$ is split,

2. $\mathcal{A}$ is strongly additive,

3. the 2-interval inclusion of factors $\mathcal{A}(E) \subset \mathcal{A}\left(E^{\prime}\right)^{\prime}$ has finite Jones index $\mu_{\mathcal{A}}$.

Here both $E \subset S^{1}$ and $E^{\prime} \equiv S^{1} \backslash E$ are the union of two proper intervals. The split and strongly additivity properties are well-studied basic properties, see Section 3.5 for their definitions, and we do not dwell on them here, cf. [13, 7, 11] and [8, 23]. If $\mathcal{A}$ is completely rational, then $\mathcal{A}(E) \subset \mathcal{A}\left(E^{\prime}\right)^{\prime}$ is obtained by a quantum double construction in [34], in particular

$$
\mu_{\mathcal{A}}=\sum_{i} d\left(\rho_{i}\right)^{2}
$$

where the sum is taken over all the irreducible sectors of $\mathcal{A}$. Every representation of $\mathcal{A}$ (on a separable Hilbert space) is Möbius covariant and decomposes into the direct sum of irreducible representations with finite statistical dimension. There are only finitely many inequivalent irreducible representations, i.e. $\mathcal{A}$ is rational, and the associated braiding is non-degenerate, i.e. the representation tensor category is modular.

At this point the problem of verifying the complete rationality of known models arises. Certain examples were discussed in [27]. As an illustration from [27], consider the case of a non-trivial finite group $G$ acting on a completely rational $\mathcal{A}$; if the fixed-point orbifold subnet $\mathcal{A}^{G}$ is also completely rational, then $\mu_{\mathcal{A}^{G}} \geq|G|^{2}$, while $\sum_{\pi \in \hat{G}} d\left(\rho_{\pi}\right)^{2}=|G|$, where the the $\rho_{\pi}$ 's are the untwisted DHR sectors of $\mathcal{A}^{G}$ [12], and this shows that twisted sectors must appear. As $\mathcal{A}$ is the initial data, one would infer the complete rationality of $\mathcal{A}^{G}$ from that of $\mathcal{A}$. By [27] $\mathcal{A}^{G}$ inherits from $\mathcal{A}$ the split property and the finiteness of the $\mu$-index. F. Xu 46 has then shown that $\mathcal{A}^{G}$ also inherits the strong additivity property and this has inspired our paper. 
We shall now show that if $\mathcal{B}$ is any conformal subnet of $\mathcal{A}$ with finite index, then $\mathcal{B}$ is completely rational iff $\mathcal{A}$ is completely rational.

As a consequence, if $\mathcal{B}$ is a cofinite subnet of $\mathcal{A}$, namely $\left[\mathcal{A}: \mathcal{B} \vee \mathcal{B}^{c}\right]<\infty$, where $\mathcal{B} \vee \mathcal{B}^{c}$ is the subnet generated by $\mathcal{B}$ and its relative commutant $\mathcal{B}^{c}$ in $\mathcal{A}$, then $\mathcal{A}$ is completely rational iff both $\mathcal{B}$ and $\mathcal{B}^{c}$ are completely rational.

The subnet $\mathcal{B}^{c}$ is called the coset subnet associated with $\mathcal{B} \subset \mathcal{A}$, as it generalizes a coset construction that plays an important rôle in the theory of Kac-Moody Lie algebras, allowing one to construct of the minimal series representations of the Virasoro algebra 19].

Coset models have been intensively studied by $\mathrm{Xu}$ in [43, 44] by operator algebraic methods. In one approach he makes use of [27] too. Thanks to his work, coset models associated with many loop group inclusions are cofinite, rational and modular, see the list in Section 3.5.1. Property 3 holds, but the validity of strong additivity was left open.

By our work in all these examples $\mathcal{B}^{c}$ is strongly additive, thus $\mathcal{B}^{c}$ turns out to be completely rational and this completes the above discussion and explains the rationality/modularity structure better.

We now comment on our proof that the complete rationality property (and also the 'split \& strongly additivity' property) for finite-index inclusions of conformal nets $\mathcal{B} \subset \mathcal{A}$ are hereditary. That 'Property $1 \& 3$ ' passes from $\mathcal{A}$ to $\mathcal{B}$ and viceversa is shown in [27]. The remaining more difficult point we have to prove is that $\mathcal{B}$ is strongly additive if $\mathcal{A}$ is split and strongly additive, see Sect. 3.5.

To this end, we have analyzed a finite-index inclusion of conformal nets $\mathcal{B} \subset \mathcal{A}$ by considering the relative superselection structure. In particular we show that, for a fixed interval $I$, every von Neumann algebra $\mathcal{R}$ intermediate between $\mathcal{B}(I)$ and $\mathcal{A}(I)$ comes from an intermediate conformal net $\mathcal{L}$ between $\mathcal{A}$ and $\mathcal{B}$ with $\mathcal{L}(I)=\mathcal{R}$.

Here we make use of a result of Watatani [42] (in the type II case), following previous work by Popa [36], and Teruya and Watatani [40] (in the type III case) to the effect that the set $\mathfrak{I}(\mathcal{N}, \mathcal{M})$ of intermediate subfactors in an irreducible inclusion of factors $\mathcal{N} \subset \mathcal{M}$ with finite Jones index $[\mathcal{M}: \mathcal{N}]$ is finite. We give a direct general proof of this result that works for arbitrary factors. This proof provides for the first time an explicit bound for the cardinality $|\mathfrak{I}(\mathcal{N}, \mathcal{M})|$ of $\mathfrak{I}(\mathcal{N}, \mathcal{M})$ which depends only on $[\mathcal{M}: \mathcal{N}]$ and implies that

$$
|\Im(\mathcal{I}, \mathcal{M})| \leq \ell^{\ell}
$$

where $\ell=[\mathcal{M}: \mathcal{N}]^{2}$. There may be better bounds taking account of further structure associated with intermediate subfactors [3, 4], see the comments at the end of Section 2.2 .

We conclude this introduction and include references to the books [1, 14, 24, 39] for basic facts on Operator Algebras and Quantum Field Theory, see also [28] for subfactors and sectors. 


\section{On subfactors and intermediate factors}

The first part of this paper is devoted to an analysis of subfactors and intermediate factors, that will be used later on.

\subsection{Some basic structure}

Let $\mathcal{N} \subset \mathcal{M}$ be an irreducible inclusion of infinite factors with finite index $[\mathcal{M}: \mathcal{N}]$. We denote by $\gamma$ the canonical endomorphism of $\mathcal{M}$ into $\mathcal{N}$ and by $\theta$ the dual canonical endomorphism $\theta \equiv \gamma \Upsilon_{\mathcal{N}}$. The Q-system associated with $\gamma$ is denoted by $(\gamma, T, S)$, namely $T \in \mathcal{M}$ and $S \in \mathcal{N}$ are the unique (up to a phase) isometries in $(\iota, \gamma)$ and $(\iota, \theta)$, where $\iota$ always denotes the identity automorphism.

Let $\left\{\left[\rho_{i}\right], i=0, \ldots N\right\}$ be the family of the irreducible sectors in the decomposition of $[\theta]$, namely

$$
[\theta]=\bigoplus_{i=0}^{N} N_{i}\left[\rho_{i}\right] .
$$

By Frobenious reciprocity for each $i$ the Hilbert space of isometries in $\mathcal{M}$ (not necessarily with right support 1)

$$
K_{i} \equiv\left\{R \in \mathcal{M}: R x=\rho_{i}(x) R \forall x \in \mathcal{N}\right\}
$$

has dimension $N_{i}$, indeed the map

$$
v \in(\rho, \theta) \rightarrow v^{*} T \in K_{i}
$$

is an anti-linear isomorphism of $(\rho, \theta)$ with $K_{i}$, whose inverse is given by

$$
X \in K_{i} \rightarrow[\mathcal{M}: \mathcal{N}] \varepsilon\left(T X^{*}\right) \in(\rho, \theta),
$$

where $\varepsilon$ is the expectation of $\mathcal{M}$ onto $\mathcal{N}$. See [34, 25] for the following.

Lemma 1. Let $\left\{R_{i, k}\right\}_{k=1}^{N_{i}}$ be an orthogonal basis of $K_{i}$ with the normalization $R_{i, k}^{*} R_{i, k}=$ $d\left(\rho_{i}\right)$. Then every $X \in \mathcal{M}$ has a unique Fourier expansion

$$
X=\sum_{i=0}^{N} \sum_{k=1}^{N_{i}} x_{i, k} R_{i, k}
$$

where the coefficients $x_{i, k}$ belong to $\mathcal{N}$, indeed $x_{i, k}=\varepsilon\left(X R_{i, k}^{*}\right)$.

Proof It is immediate that $\varepsilon\left(R_{i, k} R_{j, h}^{*}\right) \in\left(\rho_{j}, \rho_{i}\right)$, thus $\varepsilon\left(R_{i, k} R_{j, h}^{*}\right)=0$ if $i \neq j$. Let $\left\{v_{1}, \ldots v_{N_{i}}\right\}$ be an orthonormal basis of isometries for $K_{i}$, then $R_{i, k} \equiv[\mathcal{M}: \mathcal{N}]^{\frac{1}{2}} v_{k}^{*} T$ satisfy

$$
R_{i, k}^{*} R_{i, h}=[\mathcal{M}: \mathcal{N}] T^{*} v_{k} v_{h}^{*} T=\delta_{h k} d\left(\rho_{i}\right)
$$


because $v_{k} v_{h}^{*} \in(\theta, \theta)$ and $T T^{*} \in \mathcal{M}$ is the Jones projection for implementing the expectation $\varepsilon_{1}: \mathcal{N} \rightarrow \theta(M)$ so that $\varepsilon_{1} \uparrow_{(\theta, \theta)}$ is the associated trace, see [30]. Therefore

$$
\varepsilon\left(R_{i, k} R_{i, h}^{*}\right)=[\mathcal{M}: \mathcal{N}] \varepsilon\left(v_{k}^{*} T T^{*} v_{h}\right)=v_{k}^{*} v_{h}=\delta_{h k} .
$$

Now $\mathcal{M}=\mathcal{N} T$, thus $\mathcal{M}$ is generated by $\mathcal{M}=\sum_{i, k} \mathcal{N} R_{i, k}$ because $\sum K_{i}$ has right support one. By the orthogonality relations (5) every $X \in \mathcal{M}$ has the expansion given by formula (4).

Denote by $\mathfrak{I}(\mathcal{N}, \mathcal{M})$ the set of intermediate von Neumann algebras between $\mathcal{N}$ and $\mathcal{M}$. Clearly if $\mathcal{R} \in \Im(\mathcal{N}, \mathcal{M})$, then $\mathcal{R}$ is a factor and indeed both $\mathcal{N} \subset \mathcal{R}$ and $\mathcal{R} \subset \mathcal{M}$ are irreducible finite-index inclusions of factors.

Let $\mathcal{R} \in \mathfrak{I}(\mathcal{N}, \mathcal{M})$ and set $K_{i}^{\prime} \equiv K_{i} \cap \mathcal{R}$. Then $K_{i}^{\prime}$ is a Hilbert subspace of $K_{i}$, so we may choose the $R_{i, k}$ so that $\left\{R_{i, k}\right\}_{k=1}^{N_{i}^{\prime}}$ is a basis for $K_{i}^{\prime}$, where $N_{i}^{\prime}=\operatorname{dim} K_{i}^{\prime}$. We may also re-order the $\rho_{i}$ 's so that $N_{i}^{\prime}>0$ iff $i \leq N^{\prime}$ where $N^{\prime} \leq N$ is an integer.

Proposition 2. With the above notations, $X \in \mathcal{M}$ belongs to $\mathcal{R}$ iff in the expansion (4) $x_{i, k}=0$ for all $k>N_{i}^{\prime}$, namely

$$
X=\sum_{i=0}^{N^{\prime}} \sum_{k=1}^{N_{i}^{\prime}} x_{i, k} R_{i, k} .
$$

In particular $\mathcal{R}$ is generated by $\mathcal{N}$ and the $K_{i}^{\prime}$ 's.

Proof Recall that $[\mathcal{M}: \mathcal{R}]<\infty$, so there exists an expectation $\varepsilon_{\mathcal{R}}: \mathcal{M} \rightarrow \mathcal{R}$. From the definition (2) $\varepsilon_{\mathcal{R}}\left(K_{i}\right) \subset K_{i}$, thus $\left.\varepsilon_{\mathcal{R}}\right|_{K_{i}} \in B\left(K_{i}\right)$ is a norm one projection. Clearly $\varepsilon_{\mathcal{R}}\left(K_{i}\right)=K_{i}^{\prime}$ thus if $X \in \mathcal{M}$ has the expansion (四) we have

$$
\varepsilon_{\mathcal{R}}(X)=\sum_{i=0}^{N^{\prime}} \sum_{k=1}^{N_{i}^{\prime}} x_{i, k} R_{i, k},
$$

which implies the statement in the proposition.

The following theorem and its corollary are due to Watatani and Teruya-Watatani [42, 40], related results are contained in [36].

Theorem 3. $\mathfrak{I}(\mathcal{N}, \mathcal{M})$ is a finite set.

Corollary 4. Let $\mathcal{L}$ be an intermediate subfactor between $\mathcal{N}$ and $\mathcal{M}$ and $\beta: \mathfrak{G} \rightarrow$ $\operatorname{Aut}(\mathcal{M})$ a (pointwise weakly continuous) action of a connected topological group $\mathfrak{G}$ with $\beta_{g}(\mathcal{N})=\mathcal{N}, g \in \mathfrak{G}$. Then $\beta_{g}(\mathcal{L})=\mathcal{L}, g \in \mathfrak{G}$.

Proof We consider on $\Im(\mathcal{I}, \mathcal{N})$ the topology of pointwise weak convergence of the associated conditional expectations $\left(\mathcal{L}_{i} \rightarrow \mathcal{L}\right.$ iff $\varepsilon_{\mathcal{L}_{i}}(x) \rightarrow \varepsilon_{\mathcal{L}}(x)$ weakly for all $x \in \mathcal{M})$. Then $\beta$ implements a continuous action of $\mathfrak{G}$ on $\mathfrak{I}(\mathcal{M}, \mathcal{N})$. The corollary is thus immediate because any continuous action of a connected group on a discrete set is trivial.

A direct proof of Th. 3 for factors of arbitrary type will be given in next section, where we shall obtain in particular a bound for the cardinality $|\mathfrak{I}(\mathcal{N}, \mathcal{M})|$ of $\mathfrak{I}(\mathcal{N}, \mathcal{M})$. 


\subsection{A bound for the number of intermediate subfactors}

Let $\mathcal{N} \subset \mathcal{M}$ be an irreducible inclusion with finite index and denote by $\varepsilon$ the conditional expectation from $\mathcal{M}$ to $\mathcal{N}$. We shall now determine a bound for $|\mathfrak{I}(\mathcal{M}, \mathcal{N})|$. Our proof is inspired by the papers [10, 42].

We assume that there exists a faithful normal state $\omega$ on $\mathcal{N}$ (otherwise replacing it by a weight). By considering the GNS representation of $\mathcal{M}$ associated with $\tilde{\omega} \equiv \omega \cdot \varepsilon$, we may assume that $\mathcal{M}$ acts on a Hilbert space $\mathcal{H}$ with cyclic and separating vector $\Omega$ so that $\tilde{\omega}=(\Omega, \Omega)$. Then $e \equiv[\mathcal{N} \Omega]$, equal to $J e J$, is the Jones projection for $\mathcal{N} \subset \mathcal{M}$ and $\mathcal{M}_{1} \equiv\langle\mathcal{M}, e\rangle=J \mathcal{N}^{\prime} J$ is the Jones extension, where $J$ is the modular conjugation of $\mathcal{M}$ associated with $\Omega$. The projection $e \in \mathcal{N}^{\prime} \cap \mathcal{M}_{1}$ and

$$
\varepsilon(e)=\lambda \equiv[\mathcal{M}: \mathcal{N}]^{-1}
$$

Let $\mathcal{R}, \mathcal{S}$ be intermediate factors between $\mathcal{N}$ and $\mathcal{M}$ and denote by $p \equiv[\mathcal{R} \Omega]$ and $q \equiv[\mathcal{S} \Omega]$ their associated Jones projections. Note that $J p J=p, J q J=q$ and $\mathcal{R}_{1} \equiv\langle\mathcal{R}, p\rangle=J \mathcal{R}^{\prime} J$ and $\mathcal{S}_{1} \equiv\langle\mathcal{S}, q\rangle=J \mathcal{S}^{\prime} J$ are the corresponding Jones extensions and so there is a chain of inclusions

$$
\mathcal{N} \subset \mathcal{R}, \mathcal{S} \subset \mathcal{M} \subset \mathcal{R}_{1}, \mathcal{S}_{1} \subset \mathcal{M}_{1}
$$

Clearly $p \in \mathcal{R}^{\prime} \cap \mathcal{R}_{1}$ and $q \in \mathcal{S}^{\prime} \cap \mathcal{S}_{1}$, thus $p$ and $q$ both belong to $\mathcal{N}^{\prime} \cap \mathcal{M}_{1}$.

Proposition 5. If $\|p-q\|<\lambda / 2$ then $\mathcal{R}=\mathcal{S}$.

Proof We may assume $\mathcal{R} \neq \mathcal{N}$ as otherwise $p=e$, thus $q=e$ because $q \geq e$ and $\lambda \leq 1$.

As we have $[\mathcal{M}: \mathcal{N}]=[\mathcal{M}: \mathcal{R}][\mathcal{R}: \mathcal{N}]$, it follows that

$$
[\mathcal{M}: \mathcal{N}] \geq 2[\mathcal{M}: \mathcal{R}]
$$

because $[\mathcal{R}: \mathcal{N}] \geq 2\left[26\right.$. In particular $\lambda \leq 1 / 2$. Let $\varepsilon_{\mathcal{R}_{1}}$ be the expectation from $\mathcal{M}_{1}$ onto $\mathcal{R}_{1}$ and set $q^{\prime} \equiv \varepsilon_{\mathcal{R}_{1}}(q)$. Then $q^{\prime} \in \mathcal{S}^{\prime} \cap \mathcal{R}_{1}$ since obviously $q^{\prime} \in \mathcal{R}_{1}$ and if $x \in \mathcal{S}$

$$
x q^{\prime}=x \varepsilon_{\mathcal{R}_{1}}(q)=\varepsilon_{\mathcal{R}_{1}}(x q)=\varepsilon_{\mathcal{R}_{1}}(q x)=q^{\prime} x
$$

because $\mathcal{S} \subset \mathcal{R}_{1}$. Moreover $0 \leq q^{\prime} \leq 1$ and $q^{\prime} \neq 0$ because $\varepsilon_{\mathcal{R}_{1}}$ is positive and faithful. Setting $\delta=\lambda / 2$ we have

$$
\left\|p-q^{\prime}\right\|=\left\|p-\varepsilon_{\mathcal{R}_{1}}(q)\right\|=\left\|\varepsilon_{\mathcal{R}_{1}}(p-q)\right\| \leq\|p-q\|<\delta .
$$

Therefore the spectrum $\operatorname{sp}\left(q^{\prime}\right) \subset[0, \delta) \cup(1-\delta, 1]$, see Lemma 8 .

Thus the spectral projection $q^{\prime \prime} \equiv \chi_{(1-\delta, 1]}\left(q^{\prime}\right)$ is a projection in $\mathcal{S}^{\prime} \cap \mathcal{R}_{1}$ and $\left\|q^{\prime \prime}-q^{\prime}\right\|<\delta$, thus

$$
\left\|p-q^{\prime \prime}\right\| \leq\left\|p-q^{\prime}\right\|+\left\|q^{\prime}-q^{\prime \prime}\right\|<2 \delta \leq 1
$$


and this implies that $p$ and $q^{\prime \prime}$ are equivalent projections of $\mathcal{N}^{\prime} \cap \mathcal{R}_{1}$. Indeed the phase $v$ in the polar decomposition of $t \equiv p q^{\prime \prime}$ is a partial isometry in $\mathcal{N}^{\prime} \cap \mathcal{R}_{1}$ with $v^{*} v=q^{\prime \prime}, v v^{*}=p$ (see Lemma 9 below). Then we can define an isomorphism $\Phi$ of $\mathcal{S}$ into $\mathcal{R}$ by

$$
\Phi(x) p \equiv v x v^{*}, x \in \mathcal{S}
$$

as $p \mathcal{R}_{1} p=\mathcal{R} p$ [26]. Moreover $\Phi(x)=x$ for all $x \in \mathcal{N}$ because $v \in \mathcal{N}^{\prime}$.

We have the intertwining relation

$$
\Phi(x) v=v x, x \in \mathcal{S} .
$$

With $\varepsilon^{\prime}$ the conditional expectation from $\mathcal{M}_{1}$ onto $\mathcal{M}$ we then have

$$
\Phi(x) \varepsilon^{\prime}(v)=\varepsilon^{\prime}(v) x, x \in \mathcal{S},
$$

where $\varepsilon^{\prime}(v) \in \mathcal{N}^{\prime} \cap \mathcal{M}=\mathbb{C}$, thus $\varepsilon^{\prime}(v) \neq 0$ would imply that $\Phi$ is the identity on $\mathcal{S}$ and $\mathcal{S} \subset \mathcal{R}$. Reversing the rôle of $\mathcal{R}$ and $\mathcal{S}$ also $\mathcal{R} \subset \mathcal{S}$, so $\mathcal{R}=\mathcal{S}$.

To show that indeed $\varepsilon^{\prime}(v) \neq 0$ set $\lambda_{0} \equiv \varepsilon^{\prime}(p)=[\mathcal{M}: \mathcal{R}]^{-1}$ and notice that, by using Lemma 9, we have

$$
\begin{array}{r}
\left|\varepsilon^{\prime}(v)-\lambda_{0}\right| \leq\left\|\varepsilon^{\prime}(v)-\varepsilon^{\prime}(t)\right\|+\left\|\varepsilon^{\prime}(t)-\varepsilon^{\prime}(p)\right\| \leq\|v-t\|+\|t-p\| \\
=\|v-t\|+\left\|p\left(q^{\prime \prime}-p\right)\right\| \leq 2 \delta+\left\|q^{\prime \prime}-p\right\|<4 \delta=2 \lambda,
\end{array}
$$

thus $\varepsilon^{\prime}(v) \neq 0$ because $2 \lambda \leq \lambda_{0}$ by eq. (7).

Corollary 6. Let $\mathcal{N} \subset \mathcal{M}$ be an irreducible inclusion of factors. The cardinality of the set of intermediate factors between $\mathcal{N}$ and $\mathcal{M}$ is bounded by

$$
|\mathfrak{I}(\mathcal{N}, \mathcal{M})| \leq(4(n+2) \sqrt{n}+1)^{n^{2}}
$$

where $n$ is the largest integer such that $n+1 \leq[\mathcal{M}: \mathcal{N}]$.

Proof By the above proposition $|\mathfrak{I}(\mathcal{N}, \mathcal{M})|$ is dominated by the maximum number of projections $\geq e$ in $\mathcal{N}^{\prime} \cap \mathcal{M}_{1}$ whose mutual distance is $\geq \lambda / 2$. As $e$ is a minimal central projection of $\mathcal{N}^{\prime} \cap \mathcal{M}_{1}$, we can naturally embed $\mathcal{N}^{\prime} \cap \mathcal{M}_{1}$ into $\mathbb{C} \oplus \operatorname{Mat}_{m}(\mathbb{C})$, where $m$ an integer with $m+1 \leq[\mathcal{M}: \mathcal{N}]\left(m=\sum_{1}^{N} N_{i}\right.$ in eq. (四)). Indeed, as $J\left(\mathcal{N}^{\prime} \cap \mathcal{M}_{1}\right) J=\mathcal{N}^{\prime} \cap \mathcal{M}_{1}$, and Ad $J$ implements an anti-automorphism of $\mathcal{N}^{\prime} \cap \mathcal{M}_{1}$, we can assume this anti-automorphism to extend to an anti-automorphism of $\mathbb{C} \oplus$ $\operatorname{Mat}_{m}(\mathbb{C})$ preserving the two components, in other words we may assume that the Ad $J$-invariant part of $\mathcal{N}^{\prime} \cap \mathcal{M}_{1}$ is contained in $\mathbb{R} \oplus \operatorname{Mat}_{m}(\mathbb{R})$.

Thus $|\mathfrak{I}(\mathcal{N}, \mathcal{M})|$ is dominated by the maximum number of projections in $\operatorname{Mat}_{m}(\mathbb{C})$ whose mutual distance is larger than $\frac{1}{2(n+2)}$ where $n$ is the largest interger such that $n+1 \leq[\mathcal{M}: \mathcal{N}]\left(\right.$ so $\left.\lambda>\frac{1}{n+2}\right)$. Moreover, as $J p_{i} J=p_{i}$, we may regard the $p_{i}$ 's as elements of $\operatorname{Mat}_{m}(\mathbb{R})$. 
The following Lemma $\square$ with $\epsilon=\frac{1}{2(n+2)}$ then gives

$$
|\mathfrak{I}(\mathcal{N}, \mathcal{M})| \leq(4(n+2) \sqrt{m}+1)^{m^{2}} \leq(4(n+2) \sqrt{n}+1)^{n^{2}}
$$

The following lemma slightly improves [0], Lemma 2.6].

Lemma 7. Let $\epsilon>0$ and $\left\{p_{1}, p_{2}, \ldots, p_{k}\right\}$ be elements in the unit ball of $\operatorname{Mat}_{n}(\mathbb{R})$ such that $\left\|p_{i}-p_{j}\right\| \geq \epsilon$ if $i \neq j$. Then $k \leq\left(\frac{2 \sqrt{n}}{\epsilon}+1\right)^{n^{2}}$.

Proof As the uniform and Hilbert-Schmidt norms are related by $\|X\| \leq\|X\|_{H S} \leq$ $\sqrt{n}\|X\|$, the $p_{i}$ 's give vectors of norm less $\sqrt{n}$ in the Euclidean space $\mathbb{R}^{n^{2}}$ (identified with $\operatorname{Mat}_{n}(\mathbb{R})$ with the Hilbert-Schmidt norm) with mutual distance larger than $\epsilon$. Denoting by $B(r)$ the open ball of radius $r$ in $\mathbb{R}^{n^{2}}$ we then have

$$
k<\frac{\operatorname{Vol}(B(\sqrt{n}+\epsilon / 2))}{\operatorname{Vol}(B(\epsilon / 2))}=\left(\frac{2 \sqrt{n}}{\epsilon}+1\right)^{n^{2}}
$$

The following lemmata are variations of known facts (cf. e.g. [39]) and are included for convenience.

Lemma 8. Let $x$ be a positive linear operator, $0 \leq x \leq 1$, and $p$ a selfadjoint projection with $\|x-p\| \leq \delta<1 / 2$. Then $\operatorname{sp}(x) \subset[0, \delta] \cup[1-\delta, 1]$.

Proof With $\ell \in(\delta, 1-\delta)$ we have $x-\ell=(p-\ell)\left(1+(p-\ell)^{-1}(x-p)\right)$, thus $x-\ell$ is invertible if $\left\|(p-\ell)^{-1}(x-p)\right\|<1$, which is the case if $\delta\left\|(p-\ell)^{-1}\right\|<1$. This holds because $\left\|(p-\ell)^{-1}\right\|=\max \left\{\ell^{-1},(1-\ell)^{-1}\right\}<\delta^{-1}$.

Lemma 9. Let $p$ and $q$ be selfadjoint projections on a Hilbert space $\mathcal{H}$ and $t=v h$ be the polar decomposition of $t \equiv p q$. If $\|p-q\| \leq \delta<1$, then $v^{*} v=q$, $v v^{*}=p$ and $\|v-t\| \leq \delta$.

Proof As $\left\|(p-q)^{2}\right\| \leq \delta^{2}<1$, the operator $1-(p-q)^{2}$ is invertible. Thus $s \equiv p q+(1-p)(1-q)$ is also invertible, indeed $s^{-1}=\left(1-(p-q)^{2}\right)^{-1} s^{*}$, and this implies that $v$ is a partial isometry from $q$ to $p$. Then we have

$$
\begin{array}{r}
\|v-t\|=\|v-v h\|=\|v(1-h)\| \leq \|(1-h)\left\lceil_ { q \mathcal { H } } \| = \| ( 1 - \sqrt { q p q } ) \left\lceil_{q \mathcal{H}} \|\right.\right. \\
\leq \|(1-q p q)\left\lceil_{q \mathcal{H}}\|=\| q(q-p) q\|\leq\| q-p \| \leq \delta .\right.
\end{array}
$$


The bound (9) implies that

$$
|\Im(\mathcal{N}, \mathcal{M})| \leq \ell^{\ell}
$$

where $\ell=[\mathcal{M}: \mathcal{N}]^{2}$. The arguments in this section can be improved, in particular taking into account that the $p_{i}$ 's are projections in Lemma 7, leading to a better bound $|\Im(\mathcal{N}, \mathcal{M})| \leq \ell_{1}^{\ell_{2}}$ where however $\ell_{2}$ is still quadratic in the index. It would be interesting to see if a bound $|\Im(\mathcal{N}, \mathcal{M})| \leq[\mathcal{M}: \mathcal{N}]^{[\mathcal{M}: \mathcal{N}]}$ holds. This is the case of the example $\mathcal{N}=\mathcal{M}^{G}$ with $G$ is a finite group where, because of the Galois correspondence (see e.g. [25]), $|\Im(\mathcal{I}, \mathcal{M})| \leq|G| !=[\mathcal{N}: \mathcal{M}]$. We note that we have not made use of the specific form of the projections associated with intermediate subfactors and the canonical algebra they generate [3, 四.

\section{Conformal nets and subnets}

We now begin our study of conformal nets. Their subnets will be analyzed through the relative superselection structure.

\subsection{Conformal nets}

Let $\mathcal{I}$ denote the family of proper intervals of $S^{1}$, namely connected subsets of $S^{1}$ of positive measure (length) strictly less than $2 \pi$. The subnet structure is rather simple for net on the 4-dimensional Minkowski spacetime (see [9]), but this does not extend to the low-dimensional case.

A net (or precosheaf) $\mathcal{A}$ of von Neumann algebras on $S^{1}$ is a map

$$
I \in \mathcal{I} \rightarrow \mathcal{A}(I) \subset B(\mathcal{H})
$$

from $\mathcal{I}$ to von Neumann algebras on fixed a Hilbert space $\mathcal{H}$ that satisfies:

A. Isotony. If $I_{1} \subset I_{2}$ belong to $\mathcal{I}$, then

$$
\mathcal{A}\left(I_{1}\right) \subset \mathcal{A}\left(I_{2}\right) .
$$

The net $\mathcal{A}$ is called a (local) conformal net if in addition it satisfies the following properties:

B. Locality. If $I_{1}, I_{2} \in \mathcal{I}$ and $I_{1} \cap I_{2}=\varnothing$ then

$$
\left[\mathcal{A}\left(I_{1}\right), \mathcal{A}\left(I_{2}\right)\right]=\{0\}
$$

where brackets denote the commutator

\footnotetext{
3.5 .3

${ }^{1}$ The locality condition will be always assumed in this paper, with the exception of Subsection
} 
C. Conformal invariance. There exists a strongly continuous unitary representation $U$ of $\operatorname{PSL}(2, \mathbb{R})$ on $\mathcal{H}$ such that

$$
U(g) \mathcal{A}(I) U(g)^{*}=\mathcal{A}(g I), \quad g \in \operatorname{PSL}(2, \mathbb{R}), I \in \mathcal{I} .
$$

Here $\operatorname{PSL}(2, \mathbb{R})$ acts on $S^{1}$ by Möbius transformations. We shall denote also by $\alpha_{g}=\operatorname{Ad} U(g)$ the adjoint action on $B(\mathcal{H})$.

D. Positivity of the energy. The generator of the one-parameter rotation subgroup of $U$ (conformal Hamiltonian) is positive.

E. Existence of the vacuum. There exists a unit $U$-invariant vector $\Omega \in \mathcal{H}$ (vacuum vector).

We shall say that a conformal net is irreducible if $\vee_{I \in \mathcal{I}} \mathcal{A}(I)=B(\mathcal{H})$. Here the lattice symbol $\vee$ denotes the von Neumann algebra generated. We recall the following Lemma whose proof can be found in [22].

Lemma 10. Let $\mathcal{A}$ be a conformal net. The following are equivalent:

(i) $\mathcal{A}$ is irreducible;

(ii) $\Omega$ is cyclic for $\vee_{I \in \mathcal{I}} \mathcal{A}(I)$ and unique $U$-invariant;

(iii) $\Omega$ is cyclic for $\vee_{I \in \mathcal{I}} \mathcal{A}(I)$ and the local von Neumann algebras $\mathcal{A}(I)$ are factors. In this case they are $I I I_{1}$-factors (unless $\mathcal{A}(I)=\mathbb{C}$ identically).

Let $\mathcal{A}$ be an irreducible conformal net. By the Reeh-Schlieder theorem [17] the vacuum vector $\Omega$ is cyclic and separating for each $\mathcal{A}(I)$. The Bisognano-Wichmann property then holds [6, 18]: the Tomita-Takesaki modular operator $\Delta_{I}$ and conjugation $J_{I}$ associated with $(\mathcal{A}(I), \Omega), I \in \mathcal{I}$, are given by

$$
\begin{gathered}
U\left(\Lambda_{I}(2 \pi t)\right)=\Delta_{I}^{i t}, t \in \mathbb{R} \\
U\left(r_{I}\right)=J_{I},
\end{gathered}
$$

where $\Lambda_{I}$ is the one-parameter subgroup of $\operatorname{PSL}(2, \mathbb{R})$ of special conformal transformations preserving $I$ and $U\left(r_{I}\right)$ implements a geometric action on $\mathcal{A}$ corresponding, the Möbius reflection on $S^{1}$ mapping $I$ onto $I^{\prime}$, i.e. fixing the boundary points of $I$, see [6].

This immediately implies Haag duality:

$$
\mathcal{A}(I)^{\prime}=\mathcal{A}\left(I^{\prime}\right), \quad I \in \mathcal{I},
$$

where $I^{\prime} \equiv S^{1} \backslash I$. 


\subsection{Representations}

Let $\mathcal{A}$ be an irreducible local conformal net. A representation $\pi$ of $\mathcal{A}$ is a map

$$
I \in \mathcal{I} \rightarrow \pi_{I},
$$

where $\pi_{I}$ is a representation of $\mathcal{A}(I)$ on a fixed Hilbert space $\mathcal{H}_{\pi}$ such that

$$
\pi_{\tilde{I}} \uparrow_{\mathcal{A}(I)}=\pi_{I}, \quad I \subset \tilde{I} ;
$$

we shall always assume that $\pi$ is locally normal, namely $\pi_{I}$ is normal for all $I \in \mathcal{I}$, which is automatic if $\mathcal{H}_{\pi}$ is separable [39].

We shall say that a representation $\rho$ is localized in a interval $I_{0}$ if $\mathcal{H}_{\rho}=\mathcal{H}$ and $\rho_{I_{0}^{\prime}}=$ id. Given an interval $I_{0}$ and a representation $\pi$ on a separable Hilbert space, there is a representation $\rho$ unitarily equivalent to $\pi$ and localized in $I_{0}$. This is due the type $I I I$ factor property.

Let $\rho$ be a representation of $\mathcal{A}$ localized in a given interval $I_{0}$. By Haag duality $\rho$ satisfies the following properties

(a) If $I \in \mathcal{I}$ and $I \supset I_{0}$ then $\rho_{I}$ is an endomorphism of $\mathcal{A}(I)$, and $\left.\rho_{\tilde{I}}\right\rceil_{\mathcal{A}(I)}=\rho_{I}$ for all $\tilde{I} \in \mathcal{I}, \tilde{I} \supset I$

(b) If $I_{1} \in \mathcal{I}$ and $I_{1} \cap I_{0}=\varnothing$, then $\rho_{I_{1}}$ is the identity on $\mathcal{A}\left(I_{1}\right)$;

(c) If $I, I_{1} \in \mathcal{I}$ and $I \supset I_{0} \cup I_{1}$, there exists a unitary $u \in \mathcal{A}(I)$ such that the representation $I \rightarrow \rho_{I}^{\prime} \equiv u \rho_{I}(\cdot) u^{*}$ is localized in $I_{1}$ (that is to say $\rho_{I_{2}}^{\prime}$ acts identically on $\mathcal{A}\left(I_{2}\right)$ if $I_{1} \cap I_{2}=\varnothing$ for all $\left.I_{2} \in \mathcal{I}\right)$.

We now make a stereographic identification $\mathbb{R}=S^{1} \backslash\{\infty\}$ and denote by $\mathcal{I}_{0} \subset \mathcal{I}$ the family of bounded intervals of $\mathbb{R}$, namely of the intervals of $S^{1}$ whose closure do not contain the point $\infty$ of $S^{1}$.

We denote by $\mathcal{A}_{0}$ the restriction of $\mathcal{A}$ to $\mathbb{R}$ (i.e. to $\mathcal{I}_{0}$ ) and by $\mathfrak{A}_{0}$ the associated quasi-local $\mathrm{C}^{*}$-algebra $\mathfrak{A}_{0} \equiv \overline{\cup_{I \in \mathcal{I}_{0}} \mathcal{A}(I)}$ (norm closure). For a characterization of the so obtained net on $\mathbb{R}$, see [23].

Given $I_{0} \in \mathcal{I}_{0}$ a DHR endomorphism $\rho$ of $\mathcal{A}_{0}$ localized in $I_{0}$ is a map

$$
\mathcal{I}_{0} \ni I \rightarrow \rho_{I}
$$

that associates to each $I \in \mathcal{I}_{0}$ a representation $\rho_{I}$ of $\mathcal{A}(I)$ on $\mathcal{H}$ such that the above conditions $(a),(b),(c)$ hold true with $\mathcal{I}$ replaced by $\mathcal{I}_{0}$.

Clearly a DHR endomorphism determines an endomorphism of $\mathfrak{A}_{0}$, still denoted by $\rho$, such that $\rho_{I}=\left.\rho\right|_{\mathcal{A}(I)}, I \in \mathcal{I}_{0}$. The above properties $(a),(b),(c)$ are immediately expressed in terms of such endomorphism of $\mathfrak{A}_{0}$; we shall use the two descriptions interchangeably without further specifications.

Proposition 11. Let $\rho$ be a DHR endomorphism on $\mathcal{A}_{0}$ localized in an interval $I_{0} \in$ $\mathcal{I}_{0}$. There exists a unique representation $\tilde{\rho}$ of $\mathcal{A}$ extending $\rho$ and localized in $I_{0}$. 
Proof Our aim is to define consistently a representation $\rho_{I}$ of $\mathcal{A}(I)$ for every $I \in \mathcal{I}$. To this end, given $I \in \mathcal{I}$, choose $I_{1} \in \mathcal{I}_{0}, I_{1} \subset I^{\prime}$ and $L \in \mathcal{I}_{0}$ let be an interval with $L \supset I_{0} \cup I_{1}$. Take then a DHR endomorphism of $\mathcal{A}_{0}$ equivalent to $\rho$ and localized in $I_{1}, \rho^{\prime}=\operatorname{Ad} u \cdot \rho$ for some unitary $u \in \mathcal{A}(L)$. We set

$$
\tilde{\rho}_{I}(a)=u^{*} a u, a \in \mathcal{A}(I),
$$

namely $\tilde{\rho}_{I}=\operatorname{Ad} u^{*} \cdot \rho_{I}^{\prime}$. Clearly $\tilde{\rho}_{I}=\rho_{I}$ if $I \in \mathcal{I}_{0}$ and a routine checking shows that $I \in \mathcal{I} \rightarrow \tilde{\rho}_{I}$ is indeed a representation of $\mathcal{A}$.

A representation $\pi$ of $\mathcal{A}$ on a Hilbert space $\mathcal{H}_{\pi}$ is covariant if there exists a unitary representation $U_{\pi}$ of the universal covering group $\operatorname{PSL}(2, \mathbb{R})$ of $\operatorname{PSL}(2, \mathbb{R})$ on $\mathcal{H}_{\pi}$ such that

$$
\operatorname{Ad} U_{\pi}(g) \cdot \pi_{I}=\pi_{g I} \cdot \operatorname{Ad} U(g), \quad g \in \operatorname{PSL}(2, \mathbb{R})^{\tilde{y}}, I \in \mathcal{I}_{0} .
$$

Here $U$ has been lifted to $\operatorname{PSL}(2, \mathbb{R})$. $\pi$ is said to have positive energy if the generator of the rotation unitary subgroup of $U_{\pi}$ is positive.

Let $\rho$ be a representation of $\mathcal{A}$ localized in $I_{0} \in \mathcal{I}$. By a local cocycle (w.r.t. to $\rho$ ) we shall mean the assignement of an interval $I \supset I_{0}$, a symmetric neighborhood $\mathcal{U}$ of the identity of $\operatorname{PSL}(2, \mathbb{R}) \tilde{\text { such that }} I_{0} \cup g I_{0} \subset I, \forall g \in \mathcal{U}$ and a strongly continuous unitary valued map $z: g \in \mathcal{U} \rightarrow z_{\rho}(g) \in \mathcal{A}(I)$ such that

$$
\begin{gathered}
z_{\rho}(g) \in \mathcal{A}(I) \\
z_{\rho}(g h)=z_{\rho}(g) \alpha_{g}\left(z_{\rho}(h)\right), \\
\operatorname{Ad} z_{\rho}(g)^{*} \cdot \rho_{\tilde{I}}(a)=\alpha_{g} \cdot \rho_{g^{-1} \tilde{I}} \cdot \alpha_{g^{-1}}(a), a \in \mathcal{A}(\tilde{I}),
\end{gathered}
$$

for some open interval $\tilde{I}$ with $\bar{I} \subset \tilde{I}$ and all $g, h \in \mathcal{U}$ such that $I \cup g I \subset \tilde{I}$. We shall then say that $z$ is localized in $I$.

If this holds, then eq. (14) is valid for all $L \in \mathcal{I}$ :

$$
\operatorname{Ad} z_{\rho}(g)^{*} \cdot \rho_{L}(a)=\alpha_{g} \cdot \rho_{g^{-1} L} \cdot \alpha_{g^{-1}}(a), \quad a \in \mathcal{A}(L) .
$$

Indeed, if $L \supset I$ then the above equation holds by additivity [17]. Thus it holds for sub-intervals $L_{0} \subset L$. Again by additivity, the equation is then satisfied for all $L \in \mathcal{I}$.

If $\rho$ is a covariant representation of $\mathcal{A}$ localized in $I_{0}$ then for any given interval $I \supset I_{0}$ there exists a local cocycle w.r.t. $\rho$ localized in $I$. Indeed if $\mathcal{U}$ is a symmetric neighborhood of the identity of $\operatorname{PSL}(2, \mathbb{R})$ such that $I_{0} \cup g I_{0} \subset I, \forall g \in \mathcal{U}$, then by Haag duality the unitaries

$$
z_{\rho}(g) \equiv U_{\rho}(g) U(g)^{*}
$$

belong to $\mathcal{A}(I)$ for all $g \in \mathcal{U}$ and clearly verify the local cocycle property (13, 144).

Notice now that, taking $I_{0}, I \in \mathcal{I}_{0}$, a local cocycle is expressed in terms of the DHR endomorphism of $\mathcal{A}_{0}$ associated with $\rho$. The converse construction is made in the following. 
Proposition 12. Let $\rho$ be a DHR endomorphism of $\mathcal{A}_{0}$ localized in the interval $I_{0} \in$ $\mathcal{I}_{0}$ and $\tilde{\rho}$ the representation of $\mathcal{A}$ extending $\rho$ given by Proposition 11. Then $\tilde{\rho}$ is covariant iff there exists a local cocycle $z_{\rho}$ w.r.t. $\rho$ (i.e. properties (19,19,14) hold with $\mathcal{I}$ replaced by $\left.\mathcal{I}_{0}\right)$.

Proof We need only to show that $\tilde{\rho}$ is covariant if there exists a local cocycle $z_{\rho}$. By the above arguments eq. (15) holds. Now set $U_{\tilde{\rho}}(g)=z_{\rho}(g) U(g)$ for $g$ in a suitable neighborhood of the identity of $\operatorname{PSL}(2, \mathbb{R})$. Then $U_{\tilde{\rho}}$ is a local representation of $\operatorname{PSL}(2, \mathbb{R})$, hence it extends to a unitary representation of $\operatorname{PSL}(2, \mathbb{R})^{\tilde{b}}$ because $\operatorname{PSL}(2, \mathbb{R})$ is simply connected. The local covariance then gives

$$
U_{\rho}(g) \rho_{L}(a) U_{\rho}^{*}(g)=\rho_{g L}\left(\alpha_{g}(a)\right), a \in \mathcal{A}(L),
$$

for any $L \in \mathcal{I}$. The covariance then follows by the group property of $U_{\rho}$, see also [20].

Before concluding this section we recall that, if $\rho$ is a localizable representation of $\mathcal{A}$, the (statistical) dimension of $\rho$ is $d(\rho) \equiv\left[\rho_{I^{\prime}}\left(\mathcal{A}\left(I^{\prime}\right)\right)^{\prime}: \rho_{I}(\mathcal{A}(I))\right]^{\frac{1}{2}}$, independently on $I \in \mathcal{I}$, and this clearly coincides with $\left[\mathcal{A}(I): \rho_{I}(\mathcal{A}(I))\right]^{\frac{1}{2}}$ if $\rho$ is localized in $I$ [31].

If $\rho$ is Möbius covariant and $d(\rho)<\infty$, then $\rho$ has positive energy [2].

\subsection{Subnets}

Let $\mathcal{A}$ be a local irreducible conformal net of von Neumann algebras on $S^{1}$ as above and $U$ the associated unitary positive energy representation of $\operatorname{PSL}(2, \mathbb{R})$ on the vacuum Hilbert space $\mathcal{H}$.

By a conformal subnet we shall mean a map

$$
I \in \mathcal{I} \rightarrow \mathcal{B}(I) \subset \mathcal{A}(I)
$$

that associates to each interval $I \in \mathcal{I}$ a von Neumann subalgebra $\mathcal{B}(I)$ of $\mathcal{A}(I)$, which is isotone

$$
\mathcal{B}\left(I_{1}\right) \subset \mathcal{B}\left(I_{2}\right), \quad I_{1} \subset I_{2}
$$

and Möbius covariant w.r.t. the representation $U$, namely

$$
U(g) \mathcal{B}(I) U(g)^{-1}=\mathcal{B}(g I)
$$

for all $g \in \operatorname{PSL}(2, \mathbb{R})$ and $I \in \mathcal{I}$.

Let $\mathcal{H}_{\mathcal{B}}$ be the closure of $\left(\vee_{I \in \mathcal{I}} \mathcal{B}(I)\right) \Omega$ and $E$ the orthogonal projection of $\mathcal{H}$ onto $\mathcal{H}_{\mathcal{B}}$. By the Reeh-Schlieder theorem $\overline{\mathcal{B}(I) \Omega}=\mathcal{H}_{\mathcal{B}}$ for each fixed $I \in \mathcal{I}$.

Clearly $\mathcal{H}_{\mathcal{B}}$ is $U$-invariant and $\Omega$ is unique $U \uparrow_{\mathcal{H}_{\mathcal{B}}}$-invariant, thus by Lemma 10 the restriction of $\mathcal{B}$ to $\mathcal{H}_{\mathcal{B}}$ is an irreducible local conformal net on $\mathcal{H}_{\mathcal{B}}$ where $U \uparrow_{\mathcal{H}_{\mathcal{B}}}$ is the associated unitary representation of $\operatorname{PSL}(2, \mathbb{R})$.

As $\Omega$ is separating for $\mathcal{A}(I), \Omega$ is also separating for $\mathcal{B}(I), I \in \mathcal{I}$. Thus the restriction map $b \in \mathcal{B}(I) \rightarrow b \Gamma_{\mathcal{H}_{\mathcal{B}}}$ is is one-to-one, so we will often identify $\mathcal{B}$ with its restriction to $\mathcal{H}_{\mathcal{B}}$; should we need to specify, we shall talk on the net $\mathcal{B}$ on $\mathcal{H}$ or on $\mathcal{H}_{\mathcal{B}}$. Note that each $\mathcal{B}(I)$ is a factor. 
Lemma 13. For each $I \in \mathcal{I}$ there is a vacuum preserving conditional expectation $\varepsilon_{I}: \mathcal{A}(I) \rightarrow \mathcal{B}(I)$ such that $\varepsilon_{\tilde{I}} \uparrow_{\mathcal{A}(I)}=\varepsilon_{I}$ if $I \subset \tilde{I}$. Thus $\mathcal{B}$ is a standard net of subfactors in the sense of [34].

Proof By the Bisognano-Wichmann property $\mathcal{B}(I)$ is globally invariant under the modular group of $(\mathcal{A}(I), \Omega)$, hence by Takesaki's theorem there exists a conditional expectation $\varepsilon_{I}: \mathcal{A}(I) \rightarrow \mathcal{B}(I)$ given by

$$
\varepsilon_{I}(a) E=E a E, a \in \mathcal{A}(I) .
$$

As $E$ is independent of $I$, we have that $\varepsilon_{\tilde{I}} \uparrow_{\mathcal{A}(I)}=\varepsilon_{I}$ if $I \subset \tilde{I}$.

By Möbius covariance the index $[\mathcal{A}(I): \mathcal{B}(I)]$ is independent of the interval $I \in \mathcal{I}$ and will be denoted by $[\mathcal{A}: \mathcal{B}]$. The following lemma is contained in [5] with the strong additivity assumption and in [11] in the conformal case.

Lemma 14. If $[\mathcal{A}: \mathcal{B}]<\infty$ then $\mathcal{B}(I)^{\prime} \cap \mathcal{A}(I)=\mathbb{C}, I \in \mathcal{I}$.

Proof By the Bisognano-Wichmann property and the uniqueness of the vacuum the modular group of $\mathcal{A}(I)$ w.r.t. $\Omega$ acts ergodically on $\mathcal{B}(I)^{\prime} \cap \mathcal{A}(I)$, hence $\mathcal{B}(I)^{\prime} \cap \mathcal{A}(I)=$ $\mathbb{C}$ because $\mathcal{B}(I)^{\prime} \cap \mathcal{A}(I)$ is finite-dimensional.

We shall make a variation of the analysis made in [34], which is needed because our nets are not directed.

Lemma 15. Let $[\mathcal{A}: \mathcal{B}]<\infty$ and $I_{0} \subset I$. There exists a canonical endomorphism $\gamma_{I}: \mathcal{A}(I) \rightarrow \mathcal{B}(I)$ with associated Q-system $\left(\gamma_{I}, T, S\right)$ such that $T \in \mathcal{A}\left(I_{0}\right)$ and $S \in \mathcal{B}\left(I_{0}\right)$ and $\gamma_{I} \uparrow_{\mathcal{A}\left(I_{0}\right)}$ is a canonical endomorphism of $\mathcal{A}\left(I_{0}\right)$ into $\mathcal{B}\left(I_{0}\right)$.

Proof Let $\mathcal{C}\left(I_{0}\right)=\left\langle\mathcal{A}\left(I_{0}\right), E\right\rangle$ and $\mathcal{C}(I)=\langle\mathcal{A}(I), E\rangle$ be the Jones extensions and $\varepsilon_{I_{0}}^{\prime}: \mathcal{C}\left(I_{0}\right) \rightarrow \mathcal{A}\left(I_{0}\right), \varepsilon_{I}^{\prime}: \mathcal{C}(I) \rightarrow \mathcal{A}(I)$ the dual expectations. Since every $X \in \mathcal{C}\left(I_{0}\right)$ can be written as $X=\sum_{i} x_{i} E y_{i}$ with $x_{i}, y_{i} \in \mathcal{A}\left(I_{0}\right)$ [26, 35], we then have

$$
\varepsilon_{I}^{\prime}(X)=\sum_{i} x_{i} \varepsilon_{I}^{\prime}(E) y_{i}=\lambda \sum_{i} x_{i} y_{i}=\sum_{i} x_{i} \varepsilon_{I_{0}}^{\prime}(E) y_{i}=\varepsilon_{I_{0}}^{\prime}(X), X \in \mathcal{A}(I),
$$

where $\lambda \equiv[\mathcal{A}: \mathcal{B}]^{-1}$, namely $\varepsilon_{I}^{\prime} \uparrow_{\mathcal{A}\left(I_{0}\right)}=\varepsilon_{I_{0}}^{\prime}$.

Let $V \in \mathcal{C}\left(I_{0}\right)$ be an isometry $V V^{*}=E$. Then a canonical endomorphism $\gamma_{I}: \mathcal{A}(I) \rightarrow \mathcal{B}(I)$ is given by

$$
\gamma_{I}(a) E=V a V^{*} .
$$

Now

$$
T=\lambda^{-1} \varepsilon_{I}^{\prime}(V), \quad S=\lambda^{-1} \varepsilon_{I}(T),
$$

are the isometries in the Q-system for $\gamma_{I}$ and $T \in \mathcal{A}\left(I_{0}\right)$ and $S \in \mathcal{B}\left(I_{0}\right)$ by the compatibility of the expectations. 
Notice the formula

$$
\gamma_{I}(a)=\lambda^{-1} \varepsilon_{I}^{\prime}\left(V a V^{*}\right), \quad a \in \mathcal{A}(I),
$$

which is obtained applying $\varepsilon_{I}^{\prime}$ to both members of eq. (16).

Proposition 16. Let $\mathcal{A}$ be a local irreducible conformal net on $S^{1}$ and $\mathcal{B} \subset \mathcal{A}$ a conformal subnet. Given an interval $I_{0} \in \mathcal{I}$ the dual canonical endomorphism $\theta_{I_{0}} \equiv$ $\gamma_{I_{0}} \Upsilon_{\mathcal{B}\left(I_{0}\right)}$ extends to a representation $\theta$ of $\mathcal{B}$ localized in $I_{0}$.

Proof First we assume $[\mathcal{A}: \mathcal{B}]<\infty$. By Proposition [1] it is sufficient to show that $\theta_{I_{0}}$ extends to a DHR endomorphism of $\mathcal{B}$ localized in $I_{0}$ (properties $(a),(b),(c)$ with $\mathcal{I}$ replaced by $\mathcal{I}_{0}$ ). This is soon verified by applying Lemma 15. Indeed property $(a)$ is an immediate consequence of this lemma. Concerning property $(b)$ notice the formula [32

$$
\theta_{I}(b)=\lambda^{-1} \varepsilon_{I}\left(T b T^{*}\right), b \in \mathcal{B}(I),
$$

with $T$ as in Lemma 15, that follows similarly to the formula (17). Then (b) follows because $T \in \mathcal{A}\left(I_{0}\right)$.

Finally property $(c)$ is immediate by the uniqueness up to inners of the canonical endomorphism [30].

The general case can be obtained along the same lines making use of 34, Theorem 3.2] instead of Lemma 15.

Proposition 17. The representation $\theta$ of $\mathcal{B}$ on $\mathcal{H}_{\mathcal{B}}$ in Prop. 16 is unitarily equivalent to the identity representation of $\mathcal{B}$ on $\mathcal{H}$. In particular $\theta$ is covariant with positive energy.

Proof Indeed the isometry $V \in \mathcal{C}(I)$ with $V V^{*}=E$ (as in Lemma 15) satisfies the equation

$$
\theta_{\tilde{I}}(b) E=V b V^{*}, b \in \mathcal{B}(\tilde{I}),
$$

if $\tilde{I}$ is an interval containing $I$.

To show that $V$ implements the desired unitary equivalence we need to further show that the above equation (18) holds true with $\tilde{I}$ replaced with an interval $I_{1}$ not containing $I$. This is certainly true if $I_{1} \cap I=\varnothing$, because in this case $\theta$ acts trivially on $\mathcal{B}\left(I_{1}\right)$ and $V \in \mathcal{C}(I)$ commutes with $\mathcal{B}\left(I_{1}\right)$ because $\mathcal{C}(I)=\langle\mathcal{A}(I), E\rangle$ and $\mathcal{B}\left(I_{1}\right)$ commute.

So we may assume that $I_{1} \supset I^{\prime}$, extending $I_{1}$ if necessary. Choose then an interval $I_{0} \subset I$ with $I_{0} \cap I_{1}=\varnothing$. By Lemma 15 we can find a canonical endomorphism $\mathcal{A}\left(I_{0}\right) \rightarrow \mathcal{B}\left(I_{0}\right)$ with dual canonical endomorphism extending to a representation $\theta^{\prime}$ of $\mathcal{A}$ localized in $I_{0}$ with a unitary $u \in \mathcal{B}(I)$ such that $\theta^{\prime}=\operatorname{Ad} u \cdot \theta$. Then the isometry $V^{\prime}$ associated with $\theta^{\prime}$ belongs to $\mathcal{C}\left(I_{0}\right)$ and is given $V^{\prime}=u V$. Therefore

$$
\theta(b) E=u \theta^{\prime}(b) u^{*}=u V^{\prime} b V^{\prime *} u^{*}=V b V^{*}, b \in \mathcal{B}\left(I_{1}\right)
$$

as desired. 
The following corollary is a consequence of the equivalence between local and global intertwiners for a finite-index covariant representation [22].

Corollary 18. Assume $[\mathcal{A}: \mathcal{B}]<\infty$ and let $\theta$ be the representation of $\mathcal{B}$ on $\mathcal{H}_{\mathcal{B}}$ in Proposition 10. Then $\theta$ has a finite direct sum decomposition

$$
\theta=\bigoplus_{i=0}^{N} N_{i} \rho_{i}
$$

where the $\rho_{i}$ 's are positive-energy covariant irreducible representation of $\mathcal{B}$ on $\mathcal{H}_{\mathcal{B}}$ localized in $I_{0}$. Thus $\theta_{I}=\oplus_{i=0}^{N} N_{i} \rho_{i I}$ is a decomposition of the canonical endomorphism for any interval $I \supset I_{0}$.

Proof As $d(\theta)=[\mathcal{A}: \mathcal{B}]<\infty$ we may decompose $\theta$ into irreducible representations $\rho_{i}$ as above localized in $I_{0}$; moreover the $\rho_{i}$ are covariant because $\theta$ is covariant, see [22]. Then by [22, Theorem 2.3] each $\rho_{i I}$ is an irreducible endomorphism of $\mathcal{B}(I)$ if $I \supset I_{0}$.

Thus, if $[\mathcal{A}: \mathcal{B}]<\infty$ then the identity representation of $\mathcal{B}$ on $\mathcal{H}$ has finite statistical dimension. The converse is also true: as we have the inclusions $\mathcal{B}(I) \subset \mathcal{A}(I) \subset$ $\mathcal{B}\left(I^{\prime}\right)^{\prime}$, if the identity representation of $\mathcal{B}$ on $\mathcal{H}$ has finite statistical dimension, namely $\left[\mathcal{B}\left(I^{\prime}\right)^{\prime}: \mathcal{B}(I)\right]<\infty$, then $[\mathcal{A}(I): \mathcal{B}(I)]<\infty$.

Corollary 19. Assume $[\mathcal{A}: \mathcal{B}]<\infty$. With the above notations, let $K_{i} \subset \mathcal{A}\left(I_{0}\right)$ be the Hilbert spaces of isometries corresponding to $\rho_{i I_{0}}$ as in eq. (可) for the inclusion $\mathcal{B}\left(I_{0}\right) \subset \mathcal{A}\left(I_{0}\right)$. If $I$ is an interval and $I \supset I_{0}$, then $\mathcal{A}(I)$ is generated by $\mathcal{B}(I)$ and $K_{i}$ as in (4).

If $R_{i} \in K_{i}$ then $\alpha_{g}\left(R_{i}\right)=z_{\rho_{i}}(g)^{*} R_{i}$, for all $g \in \operatorname{PSL}(2, \mathbb{R})^{\sim}$ such that $g I_{0} \subset I$, where $z_{\rho_{i}}(g) \in \mathcal{B}(I)$ are unitaries in the local cocycle associated with $\rho_{i}$ (19).

Proof By Corollary 18 we have $\left(\rho_{i I}, \theta_{I}\right)=\left(\rho_{i I_{0}}, \theta_{I_{0}}\right) \subset \mathcal{B}\left(I_{0}\right)$, therefore by formula (3) and Lemma $15 K_{i} \subset \mathcal{A}\left(I_{0}\right)$ is also the Hilbert space associated with $\mathcal{B}(I) \subset \mathcal{A}(I)$ as in (四). By Lemma $\mathcal{A}(I)$ is then generated by $\mathcal{B}(I)$ and the $K_{i}$ 's.

Let $g \in \operatorname{PSL}(2, \mathbb{R})$ such that $g I_{0} \subset I$. Then $\alpha_{g} \cdot \rho_{i} \cdot \alpha_{g^{-1}}$ is localized in $I$ and $\alpha_{g} \cdot \rho_{i} \cdot \alpha_{g^{-1}}=\operatorname{Ad} z_{\rho_{i}}(g)^{*} \cdot \rho_{i}$ by formula (14) and Cor. 18, where $z_{\rho_{i}}(g) \in \mathcal{B}(I)$. Therefore $\alpha_{g}\left(K_{i}\right)=z_{\rho_{i}}(g)^{*} K_{i}$, namely $\alpha_{g}\left(R_{i}\right)=z_{\rho_{i}}(g)^{*} D(g)^{*} R_{i}$ for all $R_{i} \in K_{i}$, where $D(g) \in B\left(K_{i}\right)$. It is immediate to check that $D$ locally satisfies the cocycle property with respect to $\alpha_{g}^{\rho_{i}} \equiv \operatorname{Ad} z_{\rho_{i}}(g) \cdot \alpha_{g}$, namely $D(g h)=D(g) \alpha_{g}^{\rho_{i}}(D(h))$ for $g, h$ in a suitable neighborhood of $\operatorname{PSL}(2, \mathbb{R})^{\tilde{y}}$. But $\alpha^{\rho_{i}} \uparrow_{B\left(K_{i}\right)}$ is a finite-dimensional representation of $\operatorname{PSL}(2, \mathbb{R})^{\tilde{y}}$, thus it must be trivial because $\operatorname{PSL}(2, \mathbb{R})^{\tilde{y}}$ has no nontrivial unitary finite-dimensional representations. Thus $D$ is a local finite-dimensional unitary representation of $\operatorname{PSL}(2, \mathbb{R})^{\tilde{y}}$ on $K_{i}$, so $D$ is again trivial.

Therefore $\alpha_{g}\left(R_{i}\right)=z_{\rho_{i}}(g)^{*} R_{i}$, for all $g \in \operatorname{PSL}(2, \mathbb{R})^{\sim}$ such that $g I_{0} \subset I$ as desired. 
If $\mathcal{A}$ is a conformal net on a Hilbert space $\mathcal{H}$ and $\mathcal{B}$ is a conformal subnet, we shall set $\mathcal{B}^{\prime} \equiv\left(\vee_{L \in \mathcal{I}} \mathcal{B}(L)\right)^{\prime}$, where the commutants are taken on $\mathcal{H}$. For completeness we mention the following partial extension of Cor. 18 to the infinite index case, although it is not used in this paper.

Proposition 20. Let $\mathcal{A}$ be a local irreducible conformal net on $S^{1}$ and $\mathcal{B} \subset \mathcal{A}$ a strongly additive conformal subnet. The following are equivalent:

(i) The identity representation of $\mathcal{B}$ on $\mathcal{H}$ contains the vacuum representation of $\mathcal{B}$ with multiplicity one.

(ii) The identity representation of $\mathcal{B}$ on $\mathcal{H}$ contains the vacuum representation of $\mathcal{B}$ with finite multiplicity.

(iii) $\mathcal{B}^{c}=\mathbb{C}$, where $\mathcal{B}^{c}(I) \equiv \mathcal{B}^{\prime} \cap \mathcal{A}(I)$.

Proof $($ iii $) \Longrightarrow(i)$. By Proposition 17 we have to show that the intertwiner space between the representation $\theta$ on $\mathcal{H}_{\mathcal{B}}$ and the identity representation of $\mathcal{B}$ on $\mathcal{H}_{\mathcal{B}}$ is onedimensional. If $\theta$ is localized in $I$ then, by Haag duality, any such intertwiner belongs $\left(\theta_{I}, \iota\right)$ namely it belongs to $\mathcal{B}(I)$ and intertwines $\theta_{I}$ and the identity automorphism of $\mathcal{B}(I)$. But $\left(\theta_{I}, \iota\right)$ is one-dimensional because $\mathcal{B}(I) \subset \mathcal{A}(I)$ is an irreducible inclusion of infinite factors with a normal conditional expectation [31, 15].

$(i) \Longrightarrow(i i)$ is obvious, we show $(i i) \Longrightarrow($ iii $)$. Denote by $\pi$ the subrepresentation of the identity representation of $\mathcal{B}$ on $\mathcal{H}$ corresponding to the vacuum representation and $\mathcal{K} \subset \mathcal{H}$ the corresponding subspace. Then we have a decomposition $\mathcal{K}=\mathcal{H}_{0} \otimes \mathcal{H}_{0}^{\prime}$ and $\pi=\pi_{0} \otimes$ id where $\pi_{0}$ is irreducible and $\operatorname{dim} \mathcal{H}_{0}^{\prime}<\infty$ The representation $U$ of $\operatorname{PSL}(2, \mathbb{R})$ decomposes as $U=U_{0} \otimes U_{0}^{\prime}$ and, since $\operatorname{PSL}(2, \mathbb{R})$ has no non-trivial unitary representation, $U_{0}^{\prime}$ is the identity on $\mathcal{H}_{0}^{\prime}$. As $\Omega$ is unique $U$-invariant, we then have $\operatorname{dim} \mathcal{H}_{0}^{\prime}=1$, namely $(i)$ holds.

Thus $\mathcal{K}=\mathcal{H}_{\mathcal{B}}$ and the projection $E$ onto $\mathcal{H}_{\mathcal{B}}$ belongs to the center of $\mathcal{B}^{\prime}$. In particular if $b \in \mathcal{B}^{c}(I)$ then $b$ commutes with $E$. But $E$ implements the expectation $\varepsilon_{I}$ and $b \in \mathcal{A}(I)$, so $b$ belongs to the center of $\mathcal{B}(I)$, thus $b$ is a scalar.

\subsection{Intermediate subnets}

Let $\mathcal{A}$ be a local irreducible conformal net and $\mathcal{B}$ a conformal subnet with finite index. We now show that there exists a one-to-one correspondence between $\mathfrak{I}\left(\mathcal{B}\left(I_{0}\right), \mathcal{A}\left(I_{0}\right)\right)$ for a fixed interval $I_{0}$ and the set of intermediate conformal nets between $\mathcal{B}$ and $\mathcal{A}$.

Theorem 21. Let $I_{0}$ be a fixed interval of $S^{1}$ and $\mathcal{R}$ be an intermediate subfactor between $\mathcal{B}\left(I_{0}\right)$ and $\mathcal{A}\left(I_{0}\right)$. There exists a unique conformal subnet $\mathcal{L}$ on $\mathcal{A}$ with $\mathcal{B}(I) \subset$ $\mathcal{L}(I) \subset \mathcal{A}(I)$ and $\mathcal{L}\left(I_{0}\right)=\mathcal{R}$. 
Proof Let $\Lambda_{I}$ denote as before the one-parameter group of special conformal transformations preserving $I$. As is easily seen $\Lambda_{I}(\mathbb{R})$ is exactly the $\operatorname{subgroup}$ of $\operatorname{PSL}(2, \mathbb{R})$ of those $g$ with $g I=I$. Then $t \rightarrow \beta_{t}=\operatorname{Ad} U\left(\Lambda_{I_{0}}(t)\right) \uparrow_{\mathcal{A}\left(I_{0}\right)}$ is a one-parameter automorphism group of $\mathcal{A}\left(I_{0}\right)$ leaving $\mathcal{B}\left(I_{0}\right)$ globally invariant, hence $\beta_{t}(\mathcal{R})=\mathcal{R}$ by Corollary 4 .

Now, for $I \in \mathcal{I}$ we set

$$
\mathcal{L}(I) \equiv \alpha_{g}(\mathcal{R})
$$

where $g \in \operatorname{PSL}(2, \mathbb{R})$ is a Möbius transformation such that $I_{0}=g I$ and $\alpha_{g} \equiv \operatorname{Ad} U(g)$. $\mathcal{L}(I)$ is indeed well-defined because if $h \in \operatorname{PSL}(2, \mathbb{R})$ is any other element with $I_{0}=h I$, then $h^{-1} g I_{0}=I_{0}$, thus $h^{-1} g=\Lambda_{I_{0}}(t)$ for some $t \in \mathbb{R}$ and $\alpha_{h^{-1} g}(\mathcal{R})=\beta_{t}(\mathcal{R})=\mathcal{R}$, thus $\alpha_{g}(\mathcal{R})=\alpha_{h}(\mathcal{R})$.

As $\mathcal{L}(I) \subset \mathcal{A}(I)$ and $\mathcal{A}$ is local, $\mathcal{L}\left(I_{1}\right)$ and $\mathcal{L}\left(I_{2}\right)$ clearly commute if $I_{1} \cap I_{2}=\varnothing$. To show that $I \in \mathcal{I} \rightarrow \mathcal{L}(I)$ is a conformal net we need to check the isotony property, namely that $\mathcal{L}\left(I_{1}\right) \subset \mathcal{L}(I)$ if $I_{1} \subset I$ are intervals. By conformal invariance we may assume that $I_{1}=I_{0}$ and that $I=g_{0} I_{0}$ for some $g_{0} \in \operatorname{PSL}(2, \mathbb{R})$ and then we need to show that $\alpha_{g_{0}}(\mathcal{R}) \supset \mathcal{R}$.

Now by Corollary $19 \mathcal{A}(I)$ is generated by $\mathcal{B}(I)$ and Hilbert spaces of isometries $K_{i} \in \mathcal{A}\left(I_{0}\right)$ corresponding to the expansion (雨). Moreover

$$
\alpha_{g_{0}}\left(R_{i}\right)=z_{\rho_{i}}\left(g_{0}\right)^{*} R_{i}, \quad \forall R_{i} \in K_{i},
$$

where $z_{\rho_{i}}\left(g_{0}\right) \in \mathcal{B}(I)$ by Corollary 19. With $K_{i}^{\prime} \equiv K_{i} \cap \mathcal{R}$, by Lemma $19 \mathcal{R}$ is generated by $\mathcal{B}\left(I_{0}\right)$ and the $K_{i}^{\prime \prime}$, thus

$$
\begin{aligned}
& \alpha_{g_{0}}(\mathcal{R})=\left\{\alpha_{g_{0}}\left(\mathcal{B}\left(I_{0}\right)\right), \alpha_{g_{0}}\left(K_{i}^{\prime}\right)\right\}^{\prime \prime}=\left\{\mathcal{B}(I), z_{\rho_{i}}\left(g_{0}\right)^{*} K_{i}^{\prime}\right\}^{\prime \prime} \\
&=\left\{\mathcal{B}(I), K_{i}^{\prime}\right\}^{\prime \prime} \supset\left\{\mathcal{B}\left(I_{0}\right), K_{i}^{\prime}\right\}^{\prime \prime}=\mathcal{R}
\end{aligned}
$$

as desired.

\subsection{Complete rationality is hereditary}

To simplify notations, given two different points $\mathbf{a}, \mathbf{b}$ of $S^{1}$, we shall write $[\mathbf{a}, \mathbf{b}]$ for the closure of the set of all $z \in S^{1}$ that follow $\mathbf{a}$ and precede $\mathbf{b}$ in the counterclockwise order, and by $(\mathbf{a}, \mathbf{b})$ the interior of $[\mathbf{a}, \mathbf{b}]$. Two intervals $I_{1}, I_{2}$ will be called adjacent if there are three different points $\mathbf{a}, \mathbf{b}, \mathbf{c} \in S^{1}$ such that $\bar{I}_{1}=[\mathbf{a}, \mathbf{b}], \bar{I}_{2}=[\mathbf{b}, \mathbf{c}]$ and $\bar{I}_{1} \cup \bar{I}_{2}$ belongs to $\mathcal{I}$.

Let $\mathcal{A}$ be a conformal net on $S^{1}$. Recall that $\mathcal{A}$ is strongly additive if

$$
\mathcal{A}\left(I_{1}\right) \vee \mathcal{A}\left(I_{2}\right)=\mathcal{A}(I)
$$

if $I_{1}, I_{2}$ are adjacent intervals and $I=\bar{I}_{1} \cup \bar{I}_{2} \in \mathcal{I}$. 
$\mathcal{A}$ is split if $\mathcal{A}\left(I_{0}\right) \subset \mathcal{A}(I)$ is a split inclusion of von Neumann algebras, namely $\mathcal{A}\left(I_{0}\right) \vee \mathcal{A}(I)^{\prime}$ is naturally isomorphic to $\mathcal{A}\left(I_{0}\right) \otimes \mathcal{A}(I)^{\prime}$, if $I_{0}, I \in \mathcal{I}$ and $\bar{I}_{0}$ contained in the interior of $I$.

If $\mathcal{A}$ is split and $I_{1}, I_{2}$ are intervals with disjoint closures, then $\mathcal{A}\left(I_{1}\right) \vee \mathcal{A}\left(I_{2}\right) \simeq$ $\mathcal{A}\left(I_{1}\right) \otimes \mathcal{A}\left(I_{2}\right)$ is a factor and we shall denote by $\mu_{\mathcal{A}}$ the index of the 2 -interval inclusion $\mathcal{A}\left(I_{1}\right) \vee \mathcal{A}\left(I_{2}\right) \subset\left(\mathcal{A}\left(I_{3}\right) \vee \mathcal{A}\left(I_{4}\right)\right)^{\prime}$ where $I_{3}, I_{4}$ are the two connected components of $S^{1} \backslash\left(I_{1} \cup I_{2}\right)$.

We shall say that $\mathcal{A}$ is completely rational if $\mathcal{A}$ is split, strongly additive and the index $\mu_{\mathcal{A}}<\infty$ where the $I_{i}$ 's are intervals as above.

Lemma 22. Let $\mathcal{A}$ be an irreducible local conformal net on $S^{1}$ and $\mathcal{B} \subset \mathcal{A}$ a finiteindex conformal subnet. Then $\mathcal{A}$ is split and $\mu_{\mathcal{A}}<\infty$ iff $\mathcal{B}$ is split and $\mu_{\mathcal{B}}<\infty$.

In this case the relation $\mu_{\mathcal{B}}=[\mathcal{A}: \mathcal{B}]^{2} \mu_{\mathcal{A}}$ holds.

Proof First notice that, if $\mathcal{A}$ and $\mathcal{B}$ are split, the proof of [27, Prop. 24] shows that $[\mathcal{A}: \mathcal{B}]^{2} \mu_{\mathcal{B}}=[\mathcal{A}: \mathcal{B}]^{4} \mu_{\mathcal{A}}$, thus $\mu_{\mathcal{B}}=[\mathcal{A}: \mathcal{B}]^{2} \mu_{\mathcal{A}}$ because $[\mathcal{A}: \mathcal{B}]<\infty$. So the lemma is proved if we show the following implications:

$$
\begin{aligned}
\mathcal{A} \text { split \& } \mu_{\mathcal{A}}<\infty & \Longrightarrow \mathcal{B} \text { split }, \\
\mathcal{B} \text { split \& } \mu_{\mathcal{B}}<\infty & \Longrightarrow \mathcal{A} \text { split }
\end{aligned}
$$

Now the implication $\mathcal{A}$ split $\Longrightarrow \mathcal{B}$ split is rather immediate so, by the above comment, the first implication (20) holds.

The second implication (21) is proved in [27, Prop. 25] in a specific case, but the argument given there works in general. For the convenience of the reader we make this explicit.

Let $I_{1}, I_{2}$ be intervals with disjoint closures and $I_{3}, I_{4}$ the bounded connected components of $I_{1}^{\prime} \cap I_{2}^{\prime}$. The conditional expectation $\varepsilon_{I}: \mathcal{A}(I) \rightarrow \mathcal{B}(I)$ associated with the interval $I$, where $I$ is the interior of $\bar{I}_{1} \cup \bar{I}_{2} \cup \bar{I}_{3}$, maps $\mathcal{A}\left(I_{1}\right) \vee \mathcal{A}\left(I_{2}\right)$ onto $\mathcal{B}\left(I_{3}\right)^{\prime} \cap \mathcal{B}(I)=\left(\mathcal{B}\left(I_{3}\right) \vee \mathcal{B}\left(I_{4}\right)\right)^{\prime}$, thus

$$
\left.\varepsilon \equiv \varepsilon_{0} \cdot \varepsilon_{I}\right|_{\mathcal{A}\left(I_{1}\right) \vee \mathcal{A}\left(I_{2}\right)}
$$

is a normal faithful expectation of $\mathcal{A}\left(I_{1}\right) \vee \mathcal{A}\left(I_{2}\right)$ onto $\mathcal{B}\left(I_{1}\right) \vee \mathcal{B}\left(I_{2}\right)$, where $\varepsilon_{0}$ is a normal faithful expectation of $\left(\mathcal{B}\left(I_{3}\right) \vee \mathcal{B}\left(I_{4}\right)\right)^{\prime}$ onto $\mathcal{B}\left(I_{1}\right) \vee \mathcal{B}\left(I_{2}\right)$, that exists because $\mu_{\mathcal{B}}<\infty$.

To get the split property of $\mathcal{A}$, it will suffice to show that the above expectation $\varepsilon$ satisfies

$$
\varepsilon\left(a_{1} a_{2}\right)=\varepsilon\left(a_{1}\right) \varepsilon\left(a_{2}\right), \quad a_{i} \in \mathcal{A}\left(I_{i}\right),
$$

and $\varepsilon\left(\mathcal{A}\left(I_{i}\right)\right) \subset \mathcal{B}\left(I_{i}\right)$, as we may then compose a normal product state $\varphi_{1} \otimes \varphi_{2}$ of $\mathcal{B}\left(I_{1}\right) \vee \mathcal{B}\left(I_{2}\right) \simeq \mathcal{B}\left(I_{1}\right) \otimes \mathcal{B}\left(I_{2}\right)$ with $\varepsilon$ to get a normal product state of $\mathcal{A}\left(I_{1}\right) \vee \mathcal{A}\left(I_{2}\right)$.

Let $R_{i, k}^{(\ell)} \in \mathcal{A}\left(I_{\ell}\right), \ell=1,2$, be elements satisfying the relations (四) for the inclusion $\mathcal{B}\left(I_{i}\right) \subset \mathcal{A}\left(I_{i}\right)$, so that $\mathcal{A}\left(I_{\ell}\right)$ is generated by $\mathcal{B}\left(I_{\ell}\right)$ and the $R_{i, k}^{(\ell)}$ 's. With $a^{(\ell)} \in \mathcal{A}\left(I_{\ell}\right)$ 
we then have an expansion

$$
a^{(\ell)}=\sum_{i, k} b_{i, k}^{(\ell)} R_{i, k}^{(\ell)}, \quad b_{i, k}^{(\ell)} \in \mathcal{A}\left(I_{\ell}\right),
$$

hence

$$
a^{(1)} a^{(2)}=\sum_{i, h, j, k} b_{i, h}^{(1)} b_{j, k}^{(2)} R_{i, h}^{(1)} R_{j, k}^{(2)}
$$

so we have to show that $\varepsilon\left(R_{i, h}^{(1)} R_{j, k}^{(2)}\right)=0$ unless $i=j=0$. Now $R_{i, h}^{(1)}=u_{i, h} R_{i, h}^{(2)}$ for some unitary $u_{i, h} \in\left(\mathcal{B}\left(I_{3}\right) \vee \mathcal{B}\left(I_{4}\right)\right)^{\prime} \subset \mathcal{B}(I)$ and we have

$$
\begin{aligned}
\varepsilon\left(R_{i, h}^{(1)} R_{j, k}^{(2)}\right)=\varepsilon\left(u_{i, h} R_{i, h}^{(2)} R_{j, k}^{(2)}\right)=\varepsilon_{0}\left(u_{i, h} \varepsilon_{I}\left(R_{i, h}^{(2)} R_{j, k}^{(2)}\right)\right) & \\
& =\varepsilon_{0}\left(u_{i, h} \varepsilon_{I_{2}}\left(R_{i, h}^{(2)} R_{j, k}^{(2)}\right)\right)=\varepsilon_{0}\left(u_{i, h}\right) \varepsilon_{I_{2}}\left(R_{i, h}^{(2)} R_{j, k}^{(2)}\right) .
\end{aligned}
$$

As $\varepsilon_{0}\left(u_{i, h}\right) \in \mathcal{B}\left(I_{1}\right) \vee \mathcal{B}\left(I_{2}\right)$ is an intertwiner between irreducible endomorphisms localized in $I_{1}$ and $I_{2}$, we have $\varepsilon_{0}\left(u_{i, h}\right)=0$, thus $\varepsilon\left(R_{i, h}^{(1)} R_{j, k}^{(2)}\right)=0$, for all $i \neq 0$. If $i=0$ and $j \neq 0$, then again $\varepsilon\left(R_{i, h}^{(1)} R_{j, k}^{(2)}\right)=\varepsilon\left(R_{j, k}^{(2)}\right)=0$ because $\varepsilon_{I}\left(R_{j, k}^{(2)}\right)=\varepsilon_{I_{2}}\left(R_{j, k}^{(2)}\right)=0$.

Lemma 23. Let $\mathcal{A}$ be an irreducible local conformal net on $S^{1}$ and $\mathcal{B} \subset \mathcal{A}$ a finiteindex conformal subnet. Then $\mathcal{A}$ is strongly additive if $\mathcal{B}$ is strongly additive.

Proof Let $I_{1}, I_{2}$ be adjacent intervals with $I \equiv \bar{I}_{1} \cup \bar{I}_{2}$ and let $T \in \mathcal{A}\left(I_{1}\right)$ be the isometry in the Q-system for $\gamma_{I_{1}}$ as in Lemma 15. Then, by applying Lemma 15, $T$ is also the isometry in the Q-system associated with $\gamma_{I}$. In particular $\mathcal{A}\left(I_{1}\right)=\mathcal{B}\left(I_{1}\right) T$ and $\mathcal{A}(I)=\mathcal{B}(I) T$, thus

$$
\mathcal{A}\left(I_{1}\right) \vee \mathcal{A}\left(I_{2}\right)=\mathcal{B}\left(I_{1}\right) T \vee \mathcal{A}\left(I_{2}\right) \supset\left\{\mathcal{B}\left(I_{1}\right) \vee \mathcal{B}\left(I_{2}\right), T\right\}^{\prime \prime}=\{\mathcal{B}(I), T\}^{\prime \prime}=\mathcal{A}(I) .
$$

This concludes the proof.

Theorem 24. Let $\mathcal{A}$ be an irreducible local conformal net on $S^{1}$ and $\mathcal{B} \subset \mathcal{A}$ a conformal subnet with $[\mathcal{A}: \mathcal{B}]<\infty$. Then $\mathcal{A}$ is completely rational iff $\mathcal{B}$ is completely rational.

We postpone the proof of this theorem to Subsection 3.5.2. 


\subsubsection{Complete rationality of coset models}

We begin with the following simple lemma.

Lemma 25. Let $\mathcal{A}_{1}$ and $\mathcal{A}_{2}$ be irreducible local conformal nets on $S^{1}$. Then $\mathcal{A}_{1} \otimes \mathcal{A}_{2}$ is split (resp. strongly additive; completely rational) iff both $\mathcal{A}_{1}$ and $\mathcal{A}_{2}$ are split (resp. strongly additive; completely rational).

Proof All this can be checked directly, see [13, 27.

Let $\mathcal{A}$ be an irreducible local conformal net on $S^{1}$ and $\mathcal{B} \subset \mathcal{A}$ a conformal subnet. Then

$$
\mathcal{B}^{c}: I \in \mathcal{I} \rightarrow \mathcal{B}^{c}(I) \equiv \mathcal{B}^{\prime} \cap \mathcal{A}(I)
$$

is clearly a conformal subnet of $\mathcal{A}$ and is called the coset net associated with $\mathcal{B} \subset \mathcal{A}$, cf. [13]. Also $\mathcal{B} \vee \mathcal{B}^{c}: I \in \mathcal{I} \rightarrow \mathcal{B}(I) \vee \mathcal{B}^{c}(I)$ is then a conformal subnet of $\mathcal{A}$.

Following $\mathrm{F}$. Xu [43], we call $\mathcal{B}$ cofinite in $\mathcal{A}$ if $\left[\mathcal{A}: \mathcal{B} \vee \mathcal{B}^{c}\right]<\infty$. Indeed in [43] $\mathcal{B}$ is strongly additive so $\mathcal{B}^{c}(I)=\mathcal{B}(I)^{\prime} \cap \mathcal{A}(I)$ is the relative commutant of $\mathcal{B}(I)$ in $\mathcal{A}(I)$.

By Takesaki theorem $\mathcal{B}(I) \vee \mathcal{B}^{c}(I)$ is naturally isomorphic to the von Neumann tensor product $\mathcal{B}(I) \otimes \mathcal{B}^{c}(I)$.

Corollary 26. Let $\mathcal{A}$ be an irreducible local conformal net on $S^{1}$ and $\mathcal{B} \subset \mathcal{A}$ a cofinite conformal subnet. With the above notations, $\mathcal{A}$ is completely rational iff both $\mathcal{B}$ and $\mathcal{B}^{c}$ are completely rational.

If $\mathcal{A}$ is 'split $\mathcal{E}$ strongly additive', so are $\mathcal{B}$ and $\mathcal{B}^{c}$.

Proof It is enough to apply Theorem 24 and Lemma 25 and Proposition 34 below.

To give a first application, suppose now that the net $\mathcal{A}$ is diffeomorphism invariant. Then one can consider the conformal subnet $\mathcal{A}_{\operatorname{Vir}(c)} \subset \mathcal{A}$, which is associated with the vacuum representation of the Virasoro algebra with central charge $c>0$ see e.g. 8.

Corollary 27. Let $\mathcal{A}$ be split, strongly additive and diffeomorphism invariant. If $\mathcal{A}_{\operatorname{Vir}(c)}$ is cofinite in $\mathcal{A}$, then $c \leq 1$.

Proof If $\mathcal{A}_{\operatorname{Vir}(c)}$ is cofinite in $\mathcal{A}$ then by Cor. 26 also $\mathcal{A}_{\operatorname{Vir}(c)}$ is strongly additive, which is not the case if $c>1[8]$.

We now turn our attention to coset models. Let $G$ be simply connected semisimple compact Lie group of type $A$, i.e. $G=S U\left(N_{1}\right) \times S U\left(N_{2}\right) \times \cdots \times S U\left(N_{n}\right)$. If $H \subset G$ is a closed subgroup, there is a corresponding inclusion of loop groups $L H \subset L G$. Then the vacuum representation of $L G$ at level $k$ (see [37]) gives an inclusion of conformal nets denoted by $H \subset G_{k}$ (where $H$ may also have a suffix denoting the appearing level). Thanks to results of $\mathrm{Xu}$ [43, Corollary 3.1], see also the correction in [44], the inclusions of conformal nets associated with 
(i) $G_{k_{1}+k_{2}+\cdots+k_{m}} \subset G_{k_{1}} \times G_{k_{2}} \times \cdots G_{k_{m}}$ where the inclusion is diagonal, $k_{i} \in \mathbb{N}$, $i=1, \ldots, m$ and $G=S U(n)$,

(ii) $H_{\ell k} \subset G_{\ell}$, if $H_{k} \subset G_{1}$ is a conformal inclusion, where $k$ is the Dynkin index, $\ell \in \mathbb{N}, H$ is simple and of type $A$ and $G$ is simple,

(iii) $H \subset G_{m}$, where $H$ is the Cartan subgroup of $G$,

are all cofinite. So we have the following corollary.

Corollary 28. The coset subnets corresponding to the inclusions of nets in $(i),(i i)$, (iii) of the above Xu's list are completely rational.

Proof As the conformal net $S U(N)_{k}$ is completely rational 45 (a correct proof of the strong additivity is contained in [41]), and the subnets in Xu's list are cofinite [43, 44, it is then enough to apply Theorem 24.

It then follows from [27] that for the above coset nets the tensor category of all represenations is rational and modular, as shown in [44, and the results in [27] apply.

\subsubsection{Proof of Theorem 24}

The remaining and more difficult part to prove in Theorem 24 is that $\mathcal{A}$ split and strongly additive implies that $\mathcal{B}$ is strongly additive? In the following we thus assume that $\mathcal{A}$ is split and strongly additive and prove that $\mathcal{B}$ is strongly additive in a series of Lemmata. The starting argument is similar to the one in 46.

Lemma 29. Let $\mathcal{A}$ be an irreducible, split and strongly additive, local conformal net on $S^{1}$. If $\mathcal{B} \subset \mathcal{A}$ a conformal subnet with $[\mathcal{A}: \mathcal{B}]<\infty$ and $I_{1}$ and $I_{2}$ are adjacent intervals, $I=\bar{I}_{1} \cup \bar{I}_{2}$, then $\mathcal{A}\left(I_{1}\right) \vee \mathcal{B}\left(I_{2}\right) \subset \mathcal{A}(I)$ is a finite-index irreducible inclusion of factors.

Proof First notice that

$$
\left(\mathcal{A}\left(I_{1}\right) \vee \mathcal{B}\left(I_{2}\right)\right)^{\prime} \cap \mathcal{A}(I)=\mathcal{A}\left(I_{1}\right)^{\prime} \cap \mathcal{A}(I) \cap \mathcal{B}\left(I_{2}\right)^{\prime}=\mathcal{A}\left(I_{2}\right) \cap \mathcal{B}\left(I_{2}\right)^{\prime}=\mathbb{C},
$$

where $\mathcal{A}\left(I_{1}\right)^{\prime} \cap \mathcal{A}(I)=\mathcal{A}\left(I_{2}\right)$ because $\mathcal{A}$ is strongly additive. Thus $\mathcal{A}\left(I_{1}\right) \vee \mathcal{B}\left(I_{2}\right) \subset$ $\mathcal{A}(I)$ is an irreducible inclusion of factors.

To show that $\left[\mathcal{A}(I): \mathcal{A}\left(I_{1}\right) \vee \mathcal{B}\left(I_{2}\right)\right]<\infty$ we set $I_{1}=[\mathbf{a}, \mathbf{b}], I_{2}=[\mathbf{b}, \mathbf{c}]$ and take intervals $I_{2}^{n}=\left[\mathbf{b}_{n}, \mathbf{c}\right]$, where lenght $I_{2}^{n} \nearrow$ lenght $I_{2}$. Then, by the split property, $\mathcal{N}_{n} \equiv$ $\mathcal{A}\left(I_{1}\right) \vee \mathcal{B}\left(I_{2}^{n}\right) \subset \mathcal{M}_{n} \equiv \mathcal{A}\left(I_{1}\right) \vee \mathcal{A}\left(I_{2}^{n}\right)$ is isomorphic to $\mathcal{A}\left(I_{1}\right) \otimes \mathcal{B}\left(I_{2}^{n}\right) \subset \mathcal{A}\left(I_{1}\right) \otimes \mathcal{A}\left(I_{2}^{n}\right)$ and thus $\left[\mathcal{M}_{n}: \mathcal{N}_{n}\right]=\lambda^{-1}$, where $\lambda=[\mathcal{A}: \mathcal{B}]^{-1}$. As

$$
\mathcal{N}_{n} \nearrow \mathcal{A}\left(I_{1}\right) \vee \mathcal{B}\left(I_{2}\right), \quad \mathcal{M}_{n} \nearrow \mathcal{A}\left(I_{1}\right) \vee \mathcal{A}\left(I_{2}\right)=\mathcal{A}(I),
$$

we have $\left[\mathcal{A}(I): \mathcal{A}\left(I_{1}\right) \vee \mathcal{B}\left(I_{2}\right)\right] \leq \lambda^{-1}$ by [27, Proposition 3].

\footnotetext{
${ }^{2}$ The reader should be warned that several proofs of strong additivity for specific models in the literature are fallacious.
} 
As in Xu's proof for the group case, we consider two adjacent intervals $I_{1}, I_{2}$ and set $I=\bar{I}_{1} \cup \bar{I}_{2}$. Then $\left[\mathcal{A}(I): \mathcal{A}\left(I_{1}\right) \vee \mathcal{B}\left(I_{2}\right)\right]<\infty$, and we consider an expectation $\mu: \mathcal{A}(I) \rightarrow \mathcal{A}\left(I_{1}\right) \vee \mathcal{B}\left(I_{2}\right)$.

Then $\mathcal{R}\left(I_{2}\right) \equiv \mu\left(\mathcal{A}\left(I_{2}\right)\right)$ is contained in $\mathcal{A}\left(I_{1}\right)^{\prime} \cap \mathcal{A}(I)$ and the latter coincides with $\mathcal{A}\left(I_{2}\right)$ because $\mathcal{A}$ is strongly additive [23]. Hence $\mathcal{R}\left(I_{2}\right)$ is a von Neumann algebra and $\mathcal{B}\left(I_{2}\right) \subset \mathcal{R}\left(I_{2}\right) \subset \mathcal{A}\left(I_{2}\right)$. The following lemma is contained in 46.

Lemma 30. If $\mathcal{R}\left(I_{2}\right)=\mathcal{A}\left(I_{2}\right)$ then $\mathcal{B}$ is strongly additive.

Proof If $\mathcal{R}\left(I_{2}\right)=\mathcal{A}\left(I_{2}\right)$ then

$$
\mu(\mathcal{A}(I)) \supset \mathcal{A}\left(I_{1}\right) \vee \mathcal{R}\left(I_{2}\right)=\mathcal{A}\left(I_{1}\right) \vee \mathcal{A}\left(I_{2}\right)=\mathcal{A}(I),
$$

thus $\mu$ is the identity and $\mathcal{A}\left(I_{1}\right) \vee \mathcal{B}\left(I_{2}\right)=\mathcal{A}(I)$. We then have

$$
\mathcal{B}(I)=\varepsilon_{I}(\mathcal{A}(I))=\varepsilon_{I}\left(\mathcal{A}\left(I_{1}\right) \vee \mathcal{B}\left(I_{2}\right)\right)=\varepsilon_{I}\left(\mathcal{A}\left(I_{1}\right)\right) \vee \mathcal{B}\left(I_{2}\right)=\mathcal{B}\left(I_{1}\right) \vee \mathcal{B}\left(I_{2}\right)
$$

Lemma 31. Assume $\mathcal{R}\left(I_{2}\right)=\mathcal{B}\left(I_{2}\right)$. Given intervals $L_{0} \subset L, \bar{L}_{0} \neq \bar{L}$, and $\epsilon>0$ there exists a projection $e \in \mathcal{A}(L)$ such that

$$
\begin{gathered}
\varepsilon_{L_{0}}(a) e=e a e, \forall a \in \mathcal{A}\left(L_{0}\right) \\
(e \Omega, \Omega)>1-\epsilon .
\end{gathered}
$$

Proof As $\mathcal{B}\left(I_{2}\right)^{\prime} \cap \mathcal{A}\left(I_{2}\right)=\mathbb{C}$, there exists a unique expectation of $\mathcal{A}\left(I_{2}\right)$ onto $\mathcal{B}\left(I_{2}\right)$, thus

$$
\mu \uparrow_{\mathcal{A}\left(I_{2}\right)}=\varepsilon_{I_{2}}
$$

is the vacuum preserving conditional expectation.

In order to show the Lemma we can clearly assume that $L_{0}$ and $L$ have one common boundary point. As the Möbius group acts transitively on the family of three different points of $S^{1}$, property (22,23) does not depend on the choice of the pair $L_{0} \subset L$.

Let $e \neq 0$ be a projection in $B(\mathcal{H})$ implementing $\mu$ namely

$$
\mu(a) e=e a e, a \in \mathcal{A}(I)
$$

As $\mu$ acts identically on $\mathcal{A}\left(I_{1}\right)$, we have $e \in \mathcal{A}\left(I_{1}\right)^{\prime}=\mathcal{A}\left(I_{1}^{\prime}\right)$.

Setting $L=I_{1}^{\prime}, L_{0}=I_{2}$ we then have: $L \supset L_{0}$ are intervals with one common boundary point and there exists a non-zero projection $e \in \mathcal{A}(L)$, such that the property in formula (22) holds, i.e.

$$
e \neq 0 \& \varepsilon_{L_{0}}(a) e=e a e, \forall a \in \mathcal{A}\left(L_{0}\right) .
$$


Clearly the above property (24) is a fortiori true if we replace $L$ with a larger interval and $L_{0}$ with a smaller interval.

Set $L_{0}=[\mathbf{a}, \mathbf{b}], L=[\mathbf{a}, \mathbf{c}]$ and choose sequences of points $\mathbf{b}_{n} \in(\mathbf{a}, \mathbf{b})$ and $\mathbf{c}_{n} \in$ $(\mathbf{c}, \mathbf{a})$ in $S^{1}$, such that lenght $\left[\mathbf{a}, \mathbf{b}_{n}\right] \searrow 0$ and lenght $\left[\mathbf{a}, \mathbf{c}_{n}\right] \nearrow 2 \pi$.

As $\left[\mathbf{b}_{n}, \mathbf{c}_{n}\right]$ is an increasing sequence of intervals and $\cup_{n}\left[\mathbf{b}_{n}, \mathbf{c}_{n}\right]$ is dense in $S^{1}$, it follows that $\cup \mathcal{A}\left(\left[\mathbf{b}_{n}, \mathbf{c}_{n}\right]\right.$ ) is strongly dense in $B(\mathcal{H})$ (this is a consequence of Haag duality and the factoriality of the local von Neumann algebras). Therefore the unitaries of $\cup_{n} \mathcal{A}\left(\left[\mathbf{b}_{n}, \mathbf{c}_{n}\right]\right)$ are strongly dense in the unitaries of $B(\mathcal{H})$. Given $\epsilon>0$, then there exists an integer $n$ and a unitary $u \in \mathcal{A}\left(\left[\mathbf{b}_{n}, \mathbf{c}_{n}\right]\right)$ such that

$$
(e u \Omega, u \Omega)>1-\epsilon
$$

Replacing $L$ with $\left[\mathbf{a}, \mathbf{c}_{n}\right], L_{0}$ with $\left[\mathbf{a}, \mathbf{b}_{n}\right]$ and $e$ with $e^{\prime} \equiv u^{*} e u$, equation (23) clearly holds. But also equation (22) is satisfied because

$$
\varepsilon_{L_{0}}(a) e^{\prime}=u^{*} \varepsilon_{L_{0}}(a) e u=u^{*} e a e u=u^{*} e u a u^{*} e u=e^{\prime} a e^{\prime}, \quad a \in \mathcal{A}\left(L_{0}\right)
$$

as $u$ commutes with $\mathcal{A}\left(L_{0}\right)$.

Lemma 32. $\mathcal{R}\left(I_{2}\right) \neq \mathcal{B}\left(I_{2}\right)$ unless $\mathcal{B}=\mathcal{A}$.

Proof Let's assume $\mathcal{R}\left(I_{2}\right)=\mathcal{B}\left(I_{2}\right)$. Note that by Möbius covariance the equality $\mathcal{R}\left(I_{2}\right)=\mathcal{B}\left(I_{2}\right)$ is independent of the choice of $I_{1}, I_{2}$.

Let $I$ be a fixed interval and $I_{n}$ a decreasing sequence of intervals with a common boundary point with $I$ such that $\cap_{n} I_{n}=I$ and choose a projection $e_{n} \in \mathcal{A}\left(I_{n}\right)$ such that

$$
\varepsilon_{I}(a) e_{n}=e_{n} a e_{n}, \forall a \in \mathcal{A}(I) \&\left(e_{n} \Omega, \Omega\right)>1-\frac{1}{n} .
$$

Let $e$ be a weak limit point of $\left\{e_{n}\right\}$. Then $e \in \cap \mathcal{A}\left(I_{n}\right)=\mathcal{A}(I)$ and $e \in \mathcal{B}(I)^{\prime}$, thus $e$ is a scalar, $0 \leq e \leq 1$. As $\left(e_{n} \Omega, \Omega\right)>1-\frac{1}{n}$, we have $e=1$. Thus $e_{n} \rightarrow 1$ weakly. As the weak and strong topologies coincide on the set of selfadjoint projections, $e_{n} \rightarrow 1$ strongly. Going to the limit in eq. (25) we then have $\varepsilon_{I}(a)=a, a \in \mathcal{A}(I)$, namely $\mathcal{B}(I)=\mathcal{A}(I)$.

The assumptions in the following lemma will later be proven to be impossible.

Lemma 33. Let $\mathcal{A}$ be a local irreducible conformal net and $\mathcal{B}$ a finite-index subnet. Suppose $\mathcal{A}$ is split and strongly additive and $\mathcal{B}$ is not strongly additive. Then there exists an intermediate conformal net $\mathcal{B} \subset \mathcal{L} \subset \mathcal{A}$ such that $\mathcal{L}$ is split and strongly additive and $\mathcal{L} \neq \mathcal{A}$. 
Proof We use the above notations. By Lemma $32 \mathcal{R}\left(I_{2}\right) \neq \mathcal{B}\left(I_{2}\right)$ and Lemma 30 $\mathcal{R}\left(I_{2}\right) \neq \mathcal{A}\left(I_{2}\right)$. By Theorem 21 there exists a conformal subnet $\mathcal{R}$ intermediate between $\mathcal{B}$ and $\mathcal{A}$ such that the associated local von Neumann algebra $\mathcal{R}\left(I_{2}\right)$ is such a factor. Set $\mathcal{R}_{1} \equiv \mathcal{R}$. Replacing $\mathcal{B}$ by $\mathcal{R}_{1}$ and repeating the construction we find a factor $\mathcal{R}_{2}\left(I_{2}\right)$ between $\mathcal{R}_{1}\left(I_{2}\right)$ and $\mathcal{A}\left(I_{2}\right)$. Iterating the procedure we get a sequence of factors $\mathcal{R}_{n}\left(I_{2}\right)$, coming from a conformal subnets $\mathcal{R}_{n}$, such that

$$
\mathcal{B}\left(I_{2}\right) \subset \mathcal{R}_{1}\left(I_{2}\right) \subset \mathcal{R}_{2}\left(I_{2}\right) \subset \cdots \subset \mathcal{A}\left(I_{2}\right) .
$$

As $\left[\mathcal{A}\left(I_{2}\right): \mathcal{B}\left(I_{2}\right)\right]<\infty$, after finitely many steps the iteration stabilizes, so let $n$ be the smallest integer such that $\mathcal{R}_{n}\left(I_{2}\right)=\mathcal{R}_{n+1}\left(I_{2}\right)$. Then $n \geq 2$ by the above discussion.

By Lemma 32 we then have $\mathcal{R}_{n}\left(I_{2}\right)=\mathcal{A}\left(I_{2}\right)$. Thus $\mathcal{L} \equiv \mathcal{R}_{n-1}$ is strongly additive by Lemma 30. $\mathcal{L}$ is clearly split and, by construction, properly between $\mathcal{B}$ and $\mathcal{A}$.

Proposition 34. Let $\mathcal{A}$ be a local irreducible conformal net and $\mathcal{B}$ a finite-index subnet. If $\mathcal{A}$ is split and strongly additive then $\mathcal{B}$ is split and strongly additive.

Proof Clearly $\mathcal{B}$ is split. Suppose that $\mathcal{B}$ is not strongly additive. Then by Lemma 33 there exists a strongly additive conformal subnet $\mathcal{L}_{1}$ intermediate between $\mathcal{B}$ and $\mathcal{A}$ such that $\mathcal{L}_{1} \neq \mathcal{A}$. Again by Lemma 33 there exists a strongly additive conformal subnet $\mathcal{L}_{2}$ intermediate between $\mathcal{B}$ and $\mathcal{L}_{2}$ such that $\mathcal{L}_{2} \neq \mathcal{L}_{1}$. By iteration we find a sequence of subnets

$$
\mathcal{A} \supset \mathcal{L}_{1} \supset \mathcal{L}_{2} \supset \cdots \supset \mathcal{B}
$$

where all inclusions are proper, thus $\left[\mathcal{L}_{n+1}: \mathcal{L}_{n}\right] \geq 2$ by Jones' theorem [26]. So $[\mathcal{A}: \mathcal{B}]=\infty$ by the multiplicativity of the index [29], contrary to our assumptions.

Proof of Theorem 24 It is now sufficient to apply Lemma 22, Lemma 23 and Proposition 34.

\subsubsection{Case of a Fermi net}

Most of our analysis extends to the case of non-local Fermi conformal nets. As there are several examples of local conformal finite-index subnets of Fermi nets, we sketch here how to modify our arguments and reduce to the local situation.

In this subsection $\mathcal{A}$ is a twisted local irreducible net of von Neumann algebras on $S^{1}$, namely $\mathcal{A}$ an irreducible net satisfying all properties $\mathbf{A}$ to $\mathbf{E}$ in Section 3.1, except $\mathbf{B}$ which is now replaced by 
$\mathbf{B}^{\prime}$. Twisted locality. There exists a unitary $Z$ commuting with the unitary representation $U$ such that $Z \Omega=\Omega$ and

$$
Z \mathcal{A}\left(I^{\prime}\right) Z^{*} \subset \mathcal{A}(I)^{\prime}
$$

for all intervals $I$.

The basic results for local nets (modular structure, duality, etc.) have a version for twisted local nets, see [11].

We shall say that a conformal net $\mathcal{A}$ is a Fermi net if there exists a self-adjoint unitary $V$ on $\mathcal{H}$ such that $V \Omega=\Omega$ and $\beta(\mathcal{A}(I))=\mathcal{A}(I), I \in \mathcal{I}$, where $\beta \equiv \operatorname{Ad} V$, with canonical commutation relations: if $I_{1}, I_{2}$ are disjoint intervals then the commutator or anti-commutator

$$
\left[a_{1}, a_{2}\right]_{ \pm}=0, \quad a_{i} \in \mathcal{A}\left(I_{i}\right),
$$

if $\beta\left(a_{i}\right)= \pm a_{i}$; the commutator vanishes if one of the $a_{i}$ 's is a Bose operator $\left(\beta\left(a_{i}\right)=\right.$ $\left.a_{i}\right)$ and the anti-commutator vanishes if both the $a_{i}$ are Fermi operators $\left(\beta\left(a_{i}\right)=-a_{i}\right)$.

A conformal Fermi net satisfies twisted locality, hence twisted duality, where the unitary $Z$ is given by

$$
Z=\frac{1+i V}{1+i},
$$

in particular $Z b Z^{*}=b$, for all $b \in \vee_{I} \mathcal{B}_{b}(I)$, see [21], where we denote by $\mathcal{B}_{b}$ the Bose subnet $\mathcal{A}^{\beta}$ of $\mathcal{A}$. Note that $\left[\mathcal{A}: \mathcal{B}_{b}\right]=2$ and $\mathcal{B}_{b}$ is a local conformal net.

Lemma 35. Let $\mathcal{B}$ be a local subnet of $\mathcal{A}$. Then $\mathcal{B} \subset \mathcal{B}_{b}$.

Proof This is obvious since otherwise each $\mathcal{B}(I)$ would contain operators with nonzero Fermi part and these do not commute if they are localized in disjoint intervals.

Due to the above lemma, the results in the previous sections extend to the case of a local finite-index subnet $\mathcal{B}$ of $\mathcal{A}$ once we show them in the particular case $\mathcal{B}=\mathcal{B}_{b}$. We give here explicitly the extension of Theorem 24.

Proposition 36. Let $\mathcal{A}$ be an irreducible Fermi conformal net on $S^{1}$ and $\mathcal{B} \subset \mathcal{A}$ a local conformal subnet with $[\mathcal{A}: \mathcal{B}]<\infty$. Then $\mathcal{A}$ is split and strongly additive iff $\mathcal{B}$ is split and strongly additive.

Proof By the above discussion we may assume that $\mathcal{B}$ is the Bose subnet. We assume that $\mathcal{A}$ is split and strong additive and show that $\mathcal{B}$ strong additive, the other implications are obtained essentially as in the local case.

It is enough to show that $\mathcal{A}\left(I_{1}\right) \vee \mathcal{B}\left(I_{2}\right)=\mathcal{A}(I)$ if $I_{1}, I_{2}$ are adjacent intervals and $I=\bar{I}_{1} \cup \bar{I}_{2}$. The inclusion $\mathcal{A}\left(I_{1}\right) \vee \mathcal{B}\left(I_{2}\right) \subset \mathcal{A}(I)$ has finite index as in the local case and we assume $\mathcal{A}\left(I_{1}\right) \vee \mathcal{B}\left(I_{2}\right) \neq \mathcal{A}(I)$. We consider an expectation $\mu$ : 
$\mathcal{A}(I) \rightarrow \mathcal{A}\left(I_{1}\right) \vee \mathcal{B}\left(I_{2}\right)$. Let $u_{i} \in \mathcal{A}\left(I_{i}\right)$ be Fermi unitaries. Then $\operatorname{Ad} u_{2}$ implements an automorphism of $\mathcal{B}\left(I_{2}\right)$, acts trivially on $\mathcal{B}\left(I_{1}\right)$ and $\operatorname{Ad} u_{2}\left(u_{1}\right)=-u_{1}$, therefore $\operatorname{Ad} u_{2}$ implements an automorphism of $\mathcal{A}\left(I_{1}\right) \vee \mathcal{B}\left(I_{2}\right)$. As $\mathcal{A}(I)$ is generated by $\mathcal{A}\left(I_{1}\right) \vee \mathcal{B}\left(I_{2}\right)$ and $u_{2}$, it follows that $\mathcal{A}(I)$ is the crossed product of $\mathcal{A}\left(I_{1}\right) \vee \mathcal{B}\left(I_{2}\right)$ by $\operatorname{Ad} u_{2}$, thus $\mu$ acts trivially on $\mathcal{A}\left(I_{1}\right)$ and $\mu\left(u_{2}\right)=0$, so $\mu\left(\mathcal{A}\left(I_{2}\right)\right)=\mathcal{B}\left(I_{2}\right)$.

Let $e \neq 0$ be a projection implementing $\mu$. Then $e \in Z \mathcal{A}\left(I_{1}^{\prime}\right) Z^{*}$. Arguing as in the local case $e \in \mathcal{A}\left(I_{2}\right)^{\prime} \cap Z \mathcal{A}\left(I_{2}\right) Z^{*}$, but

$$
\begin{aligned}
\mathcal{A}\left(I_{2}\right)^{\prime} \cap Z \mathcal{A}\left(I_{2}\right) Z^{*}= & Z\left(Z^{*} \mathcal{A}\left(I_{2}\right)^{\prime} Z \cap \mathcal{A}\left(I_{2}\right)\right) Z^{*} \\
& \subset Z\left(Z^{*} \mathcal{B}\left(I_{2}\right)^{\prime} Z \cap \mathcal{A}\left(I_{2}\right)\right) Z^{*}=Z\left(\mathcal{B}\left(I_{2}\right)^{\prime} \cap \mathcal{A}\left(I_{2}\right)\right) Z^{*}=\mathbb{C} .
\end{aligned}
$$

so $e=1$ and $\mu$ is trivial, which contradicts the assumption $\mathcal{A}\left(I_{1}\right) \vee \mathcal{B}\left(I_{2}\right) \neq \mathcal{A}(I)$.

Remark. In this paper the positivity of the energy has been used only indirectly, essentially to entail the Reeh-Schlieder property and the factoriality of the local algebras. Thus our results extend to the case of conformal nets on $S^{1}$ with the above properties, without assuming the positivity of the conformal Hamiltonian. We encounter this situation if we consider a local conformal net $\mathcal{A}$ on the $1+1$-dimensional Minkowski spacetime and look at the corresponding time zero net $\mathcal{A}_{t=0}$. In particular, if $\mathcal{A}$ is a finite-index local extension of a $1+1$-dimensional chiral net $\mathcal{A}_{1} \otimes \mathcal{A}_{2}$, as discussed in [38], then $\mathcal{A}_{t=0}$ is split and strongly additive iff both $\mathcal{A}_{1}$ and $\mathcal{A}_{2}$ are split and strongly additive.

Acknowledgements. This work began while the author was visiting the Mathematical Sciences Research Institute, Berkeley, in November-December 2000, during the Operator Algebra program and he wishes to thank the organizers for the kind invitation and the warm hospitality. He also thanks M. Müger, V. Toledano and F. Xu for stimulating conversations and S. Carpi and R. Conti for critical reading of the manuscript.

\section{References}

[1] H. Araki, "Mathematical Theory of Quantum Fields", International series of monographs on Physics 101, Oxford University Press 1999.

[2] P. Bertozzini, R. Conti \& R. Longo, Covariant sectors and positivity of the energy, Commun. Math. Phys. 141 (1998), 471-492.

[3] D. Bisch, A note on intermediate subfactors, Pacific J. Math. 163 (1994), 201-216.

[4] D. Bisch \& V.F.R. Jones, Algebras associated to intermediate subfactors, Invent. Math. 128 (1997), 89-157.

[5] J. Böckenhauer \& D. E. Evans, Modular invariants, graphs and $\alpha$-induction for nets of subfactors. I, II \& III, Commun. Math. Phys. 197 (1998), 361-386, 200 (1999), 57-103 \& 205 (1999), 183-228.

J. Böckenhauer, D. E. Evans \& Y. Kawahigashi, Longo-Rehren subfactors arising from $\alpha$ induction, Publ. RIMS 31 (2001), 1-35. 
[6] R. Brunetti, D. Guido \& R. Longo, Modular structure and duality in conformal quantum field theory, Commun. Math. Phys. 156 (1993), 201-219.

[7] D. Buchholz \& C. D'Antoni, Phase space properties of charged fields in theories of local observables, Rev. Math. Phys. 7 (1995), 527-557.

[8] D. Buchholz \& H. Schulz-Mirbach, Haag duality in conformal quantum field theoery, Rev. Math. Phys. 2 (1990), 105.

[9] R. Conti, S. Doplicher \& J.E. Roberts, Superselection theory for subsystems, Commun. Math. Phys. 218 (2001), 263-281.

S. Carpi \& R. Conti, Classification of subsystems for local nets with trivial superselection structure, Commun. Math. Phys. 217, (2001), 89-106.

[10] E. Christensen, Subalgebras of a finite algebra, Math. Ann. 243 (1979), 17-29.

[11] C. D'Antoni, F. Radulescu \& R. Longo, Conformal nets, maximal temperature and models from free probability, J. Operator Theory 45, 195-208 (2001).

[12] S. Doplicher, R. Haag \& J.E. Roberts, Local observables and particle statistics. I $\&$ II, Commun. Math. Phys. 23, 199-230 (1971) \& 35, 49-85 (1974).

[13] S. Doplicher \& R. Longo, Standard and split inclusions of von Neumann algebras, Invent. Math. 73 (1984), 493-536.

[14] D. E. Evans \& Y. Kawahigashi, "Quantum Symmetries on Operator Algebras", Oxford University Press, 1998.

[15] F. Fidaleo \& T. Isola, The canonical endomorphism for infinite index inclusions, Z. Anal. und ihre Anwendungen 18 (1999), 47-66.

[16] K. Fredenhagen, Generalization of the theory of superselection sectors, in "The algebraic theory of superselection sectors" D. Kastlers ed. World Scientific 1990.

[17] K. Fredenhagen \& M. Jörß, Conformal Haag-Kastler nets, pointlike localized fields and the existence of operator product expansion, Commun. Math. Phys. 176 (1996), 541.

[18] J. Frölich \& F. Gabbiani, Operator algebras and conformal field theory, Commun. Math. Phys. 155 (1993), 569-640.

[19] P. Goddard, A. Kent \& D. Olive, Unitary representations of the Virasoro and super-Virasoro algebras, Commun. Math. Phys. 103 (1986), 105-119.

[20] D. Guido \& R. Longo, Relativistic invariance and charge conjugation in quantum field theory, Commun. Math. Phys. 148 (1992), 521-551.

[21] D. Guido \& R. Longo, An algebraic spin and statistics theorem, Commun. Math. Phys. 172 (1995), 517-533 .

[22] D. Guido \& R. Longo, The conformal spin and statistics theorem, Commun. Math. Phys. 181 (1996) 11.

[23] D. Guido, R. Longo \& H.W. Wiesbrock, Extensions of conformal nets and superselection structure, Commun. Math. Phys. 192 (1998), 217-244.

[24] R. Haag "Local Quantum Physics", Springer-Verlag (1996).

[25] M. Izumi, R. Longo \& S. Popa, A Galois correspondence for compact groups of automorphisms of von Neumann algebras with a generalization to Kac algebras, J. Funct. Anal. 10 (1998), $25-63$.

[26] V. F. R. Jones, Index for subfactors, Invent. Math. 72 (1983), 1-25. 
[27] Y. Kawahigashi, R. Longo \& M. Müger, Multi-interval subfactors and modularity of representations in conformal field theory, Commun. Math. Phys. 219 (2001), 631-669.

[28] H. Kosaki, "Type III Factors and Index Theory", Res. Inst. of Math., Lect. Notes 43, Seoul Nat. Univ. 1998.

[29] H. Kosaki \& R. Longo, A remark on the minimal index of subfactors, J. Funct. Anal. 107 (1992), 458-470.

R. Longo, Minimal index and braided subfactors, J. Funct. Anal. 109 (1992), 98-112.

[30] R. Longo, Simple injective subfactors, Adv. in Math. 63 (1986), 152-171.

[31] R. Longo, Index of subfactors and statistics of quantum fields. I E II, Commun. Math. Phys. 126 (1989) 217-247 \& 130 (1990), 285-309.

[32] R. Longo, A duality for Hopf algebras and for subfactors. I, Commun. Math. Phys. 159 (1994), $133-150$.

[33] R. Longo, An analogue of the Kac-Wakimoto formula and black hole conditional entropy, Commun. Math. Phys. 186 (1997), 451-479.

[34] R. Longo \& K.-H. Rehren, Nets of subfactors, Rev. Math. Phys. 7 (1995), 567-597.

[35] M. Pimsner \& S. Popa, Entropy and index for subfactors, Ann. Scient. Éco. Norm. Sup. 19 (1986), 57-106.

[36] S. Popa, Correspondences, INCREST manuscript, 1986.

[37] A. Pressley \& G. Segal, "Loop Groups", Oxford Science publ., Oxford 1986

[38] K.-H. Rehren, Canonical tensor product subfactors, Commun. Math. Phys. 211 (2000), 395-406. K.-H. Rehren, Chiral observables and modular invariants, Commun. Math. Phys. 208 (2000), 689-712.

[39] M. Takesaki, "Theory of Operator Algebras", I Springer-Verlag 1979; II in preparation.

[40] T. Teruya \& Y. Watatani, Lattices of intermediate subfactorsfor type III factors, Arch. Math. 68 (1997), 454-463.

[41] V. Toledano, "Fusion of positive energy representations of $\operatorname{LSpin}_{2 n}$ ", Ph.D. dissertation, Univ. of Cambridge, 1997.

[42] Y. Watatani, Lattices of intermediate subfactors, J. Funct. Anal. 140 (1996), 312-334.

[43] F. Xu, Algebraic coset conformal field theory, Commun. Math. Phys. 211 (2000), 1-43.

F. Xu, Algebraic coset conformal field theory II, Publ. RIMS 35 (1999), 795-824.

[44] F. Xu, On a conjecture of Kac-Wakimoto, Publ. RIMS 37 (2001), 165-190.

[45] F. Xu, Jones-Wassermann subfactors for disconnected intervals, Commun. Contemp. Math. 2 (2000), 307-347.

[46] F. Xu, Algebraic orbifold conformal field theory, in "Mathematical Physics in Mathematics and Physics", R. Longo ed., Fields Institute Communications Vol. 30, Amer. Math. Soc., Providence RI. 